\title{
Virtual endomorphisms of nilpotent groups
}

\author{
Adilson Berlatto and Said Sidki*
}

\begin{abstract}
A virtual endomorphism of a group $G$ is a homomorphism $f: H \rightarrow G$ where $H$ is a subgroup of $G$ of finite index $m$. The triple $(G, H, f)$ produces a state-closed (or, selfsimilar) representation $\varphi$ of $G$ on the 1-rooted $m$-ary tree. This paper is a study of properties of the image $G^{\varphi}$ when $G$ is nilpotent. In particular, it is shown that if $G$ is finitely generated, torsion-free and nilpotent then $G^{\varphi}$ has solvability degree bounded above by the number of prime divisors of $m$.
\end{abstract}

Mathematics Subject Classification (2000). Primary 20E08, $20 \mathrm{~F} 18$.

Keywords. Virtual endomorphisms, nilpotent groups, automorphisms of trees, state-closed representations.

\section{Introduction}

A virtual endomorphism of a group $G$ is a homomorphism $f: H \rightarrow G$ where $H$ is a subgroup of $G$ of finite index $m$. A recursive construction using $f$ produces a so called state-closed (or, self-similar) representation of $G$ on a 1-rooted regular $m$-ary tree. The kernel of this representation is the maximal subgroup $K$ of $H$ which is both normal in $G$ and is $f$-invariant, in the sense that $K^{f} \leq K$; it is called the $f$-core $(H)$.

The notion of virtual endomorphisms of groups is not recent. It already appeared in 1969, in M. Shub's [9], in connection with endomorphisms of compact differentiable manifolds. State-closed groups were introduced in [10], justified by the fact that the Grigorchuk 2-group, the Gupta-Sidki $p$-groups, the affine group $\mathbb{Z}^{n} \operatorname{GL}(n, \mathbb{Z})[2]$, as well as an automata group of Aleshin - claimed to be free in [1] - satisfied such a condition. State-closed representations of groups on the binary tree were studied in some depth in [7] and dynamical aspects of these were developed by Nekrashevych into a far-reaching theory in [6].

The question of existence of finite-state, state-closed representations of certain groups, especially of free groups, stimulated a number of interesting constructions.

\footnotetext{
* The second author thanks Laurent Bartholdi for hospitality at École Polytechnique Fédérale - Lausanne during November 2005 and acknowledges support from the Brazilian CNPq and Finatec.
} 
Glasner and Mozes [4] used ideas from homogeneous tree lattices to obtain such a representation of a free group of rank 14 acting on the 6-tree. This was followed by a construction by Muntyan and Savchuk (see [6], 1.10.3) for the free group of rank 2 on the 6-tree. More recently, M. Vorobets and Y. Vorobets [12] have produced a proof that a group defined on the binary tree, related to the one proposed initially by Aleshin, is indeed free of rank 3.

The present paper extends the results on free abelian state-closed groups in [7] to finitely generated nilpotent state-closed groups. The main emphasis though is on the subclass of torsion-free groups - following P. Hall's notation, these are ₹-groups, or $\mathfrak{T}_{c}$-groups when the nilpotency class is $c$.

We refer to the data $\{G, H \leq G, f: H \rightarrow G,[G: H]=m\}$ as a triple $(G, H, f)$ of degree $m$. If the $f$-core $(H)$ is the trivial subgroup then $f$ and the triple $(G, H, f)$ are called simple and when $f$ is a simple epimorphism it is called recurrent. If the only $f$-invariant subgroup of $G$ is the trivial subgroup then $f$ and the triple $(G, H, f)$ are called strongly simple. To give an example of a strongly simple triple, we let $G$ be the free nilpotent group $F(c, d)$ of class $c$, freely generated by $x_{i}(1 \leq i \leq d)$, $H=\left\langle x_{i}^{n} ; 1 \leq i \leq d\right\rangle$ where $n$ is a fixed integer greater than 1 and let $f$ be the extension of the map $x_{i}^{n} \rightarrow x_{i}(1 \leq i \leq d)$. For another example, consider the group $G$ of lower triangular matrices $\left(\begin{array}{lll}1 & 0 & 0 \\ a & 1 & 0 \\ c & b & 1\end{array}\right)$ with integer entries, $H$ its subgroup of index 4 , formed by the matrices $\left(\begin{array}{ccc}1 & 0 & 0 \\ 2 a & 1 & 0 \\ 2 c & b & 1\end{array}\right)$ and define

$$
f:\left(\begin{array}{ccc}
1 & 0 & 0 \\
2 a & 1 & 0 \\
2 c & b & 1
\end{array}\right) \rightarrow\left(\begin{array}{ccc}
1 & 0 & 0 \\
b & 1 & 0 \\
-c & a & 1
\end{array}\right)
$$

We review state-closed groups and representations in Section 2 and illustrate how to produce concretely state-closed nilpotent groups. Moreover, we prove that stateclosed abelian groups are small, in the sense that their centralizers in the group of automorphisms of the tree coincide with their topological closure in this last group.

If $G$ is an abelian group then naturally, $\operatorname{ker}(f) \leq f$-core $(H)$ for any triple $(G, H, f)$. The relationship between $\operatorname{ker}(f)$ and the $f$-core $(H)$ for general nilpotent groups is established in Section 4.

Theorem 1. Let $G$ be a nilpotent group, $H$ a subgroup of finite index $m$ in $G$, $f \in \operatorname{Hom}(H, G)$ and $L=f$-core $(H)$. Then $\operatorname{ker}(f) \leq \sqrt[H]{L}$, the isolator of $L$ in $H$.

A group $G$ is said to be to be compressible provided every subgroup $H$ of finite index in $G$ contains a subgroup $K$ isomorphic to $G$. It was shown by G. S. Smith in [11] that $\mathfrak{T}_{c}$-groups are compressible when $c \leq 2$. We extend this result to strongly simple triples in Subsection 5.1 as follows. 
Theorem 2. Let $G$ be a $\mathfrak{\Upsilon}_{c}$-group with $c \leq 2$ and let $H$ be a subgroup of finite index in $G$. Then there exists a subgroup $K$ of finite index in $H$, which admits a strongly simple epimorphism $f: K \rightarrow G$.

More on compressibility and co-Hopfianity questions concerning $\mathfrak{T}_{c}$-groups can be found in [3].

Given an integer $m>1$, let $l(m)$ be the number of prime divisors of $m$ (counting multiplicities) and $a(m)$ the largest exponent of the prime divisors of $m$. Denote by $c(G)$ the nilpotency class of $G$, by $s(G)$ the derived length of $G$ and by $d(G)$ the minimum number of generators of $G$.

If $G$ is a finitely generated nilpotent group and $H$ a subgroup of $G$ of index $m$ then it is well known that $\bar{G}=\frac{G}{\operatorname{core}(H)}$ is finite and $c(\bar{G}) \leq a(m)$. We find in Subsection 5.2 such limitations for $s(G)$ and $c(G)$ with respect to $l(m)$.

Theorem 3. Let $G$ be $a$ 霍-group and $H$ a subgroup of finite index $m$ in $G$. If $f: H \rightarrow G$ is simple then $s(G) \leq l(m)$. If $f$ is strongly simple then $c(G) \leq l(m)$.

The restriction on $G$ is striking, given that $\mathfrak{I}_{c}$-groups have faithful finite-state representations on the binary tree (that is, $m=2$ ) for any $c \geq 0$ (see [7]). To show that in the first part of the theorem $s(G)$ cannot be replaced by $c(G)$, we construct in Subsection 5.4 an ascending sequence of simple triples $\left(G_{n}, H_{n}, f_{n}\right)$ where the groups $G_{n}$ are metabelian ₹-groups with $d\left(G_{n}\right)=2, c\left(G_{n}\right)=n,\left[G_{n}: H_{n}\right]=4$. Using another sequence of examples, we show that the limit in the second part of the theorem is satisfactory.

It is important to observe that no such limitations exist for groups of prime power order. For let $p$ be a fixed prime number, $G$ be the $s$-iterated wreath product $W_{s}=$ $\left(\left(\left(C_{p}\right.\right.\right.$ wr $\left.\ldots\right)$ wr $\left.) C_{p}\right)$ wr $C_{p}, H$ its base subgroup and $\pi_{1}$ the projection of $H$ on its 1st coordinate. Then $[G: H]=p$ and $\left(G, H, \pi_{1}\right)$ is strongly simple, yet $G$ has nilpotency class $p^{s}$ and derived length $s+1$.

In Section 6 we prove the following divisibility relation between indices of subgroups.

Theorem 4. Let $G$ be a finitely generated nilpotent group, $H$ a subgroup of $G$ of finite index $[G: H]=m, f: H \rightarrow G$ a monomorphism and $\left[G: H^{f}\right]=$ $m^{\prime}$. Furthermore, let $U$ be a subgroup of $H$ and let $V=\left\langle U, U^{f}\right\rangle$. Suppose that $[V: U]=l$ and $\left[V: U^{f}\right]=l^{\prime}$ are finite. Then there exist integers $m_{1}\left|m, m_{1}^{\prime}\right| m^{\prime}$ such that $l m_{1}^{\prime}=l^{\prime} m_{1}$.

As an application, we obtain

Theorem 5. Let $G$ be a $\mathfrak{\Upsilon}$-group, $H$ a subgroup of $G$ of finite index $m$ which is a square-free integer and let $f: H \rightarrow G$ be a simple epimorphism. Then $G$ is abelian. 
The combination of conditions $a(m)=1$ and $f$ being a simple epimorphism (recurrent) in the above theorem produced $s(G)=1$; that is, $s(G)=a(m)$. This raises the question about possible improvements of the bound $l(m)$ in Theorem 3, under different types of conditions. Another question concerns the impact of the combination state-closed and finite-state would have on $\mathfrak{\mathcal { I }}$-groups. For simple triples $(G, H, f)$ where $G$ is free abelian group of finite rank and $[G: H]=m=2$, it was shown in [7] (see also, [6], Sec. 2.12) that the roots of the characteristic polynomial of $f$ lie in the interior of the unit circle.

We thank the referee for a very careful reading of our paper, for a number of positive suggestions and for providing the explicit formula in Section 5.4.

\section{State-closed groups and representations}

Let $Y$ be a non-empty set, $P(Y)$ the group of permutations of $Y$ and let $\mathcal{T}(Y)$ be 1-rooted tree indexed by the free monoid $Y^{*}$ generated by $Y$. Then the group of automorphisms $\mathcal{A}=\operatorname{Aut}(\mathcal{T}(Y))$ of the tree is isomorphic to the semidirect product of $\mathcal{A}^{Y}$ by $P(Y)$ and under this identification we have the decomposition $\mathcal{A}=\left(\mathcal{A}^{Y}\right) P(Y)$. Thus an $\alpha \in \mathcal{A}$ is represented as $\alpha=\left(\alpha_{y} \mid y \in Y\right) \alpha^{\sigma}$ where $\alpha_{y} \in \mathcal{A}$ and $\sigma \in \operatorname{Hom}(\mathcal{A}, P(Y))$.

The set of states of $\alpha$ is $Q(\alpha)=\left\{\alpha_{u} \mid u \in Y^{*}\right\}$. For $G \leq \mathcal{A}$, let $\operatorname{stab}_{G}(i)$ denote the subgroup of $G$ formed by elements which leave the $i$ th level vertices fixed. The group $\mathcal{A}$ is the inverse limit of its quotients $\frac{\mathcal{A}}{\operatorname{stab}_{\mathcal{A}}(i)}$ and as such becomes a topological group. Also, for $u \in Y^{*}$ of length $i$, let $\pi_{u}: \operatorname{stab}_{G}(i) \rightarrow \mathcal{A}$ be the projection map on the $u$ th coordinate. A subgroup $G$ of $\mathcal{A}$ is state-closed provided that $Q(\alpha)$ is a subset of $G$ for all $\alpha \in G$ and is finite-state if $Q(\alpha)$ is finite for all $\alpha \in G$.

On fixing $y \in Y$, we obtain the triple $\left(G, \operatorname{stab}_{G}(y), \pi_{y}\right)$. In the other direction, given a triple $(G, H, f)$, we represent the group $G$ on the right cosets of $H$, keeping track of the factor sets, as in Schreier's theorem. Then we use $f$ to repeat this process down a chain of subgroups. In the limit, this produces a state-closed representation of $G$ on a rooted tree of degree [ $G: H]$ as follows.

Theorem 6. Let $G$ be a group, $H$ a subgroup of $G$, and $Y$ a right transversal of $H$ in $G$. Let $\sigma$ be the permutation representation of $G$ on $Y$; for $g \in G, y \in Y$, write $g^{\sigma}: H y \rightarrow H y g=H y^{\prime}, y^{\prime} \in Y$ and $y^{\prime}=(y)^{g^{\sigma}}$. Also let $\mathcal{T}(Y)$ be a 1-rooted tree indexed by the free monoid $Y^{*}$ and let $f \in \operatorname{Hom}(H, G)$. Then the quadruple $(G, H, Y, f)$ provides a representation $\varphi$ of $G$ into the automorphism group of the tree $\mathcal{T}(Y)$ defined by

$$
g^{\varphi}=\left\{\left(y g \cdot\left(y^{g^{\sigma}}\right)^{-1}\right)^{f \varphi} \mid y \in Y\right\} g^{\sigma} .
$$

Furthermore, $\operatorname{ker}(\varphi)=f$-core $(H)$. 
To dispel difficulties with the notation in the above formula, we offer a simple example. Let $G$ be the additive group of integers $\mathbb{Z}, H=2 \mathbb{Z}$, and $Y=\{0,1\}$; then $\sigma: 0 \leftrightarrow 1$. Define $f: 2 \mathbb{Z} \rightarrow \mathbb{Z}$ by $2 n \rightarrow n$. Then $1^{\varphi}=\left(0^{\varphi}, 1^{\varphi}\right) \sigma$, which is none other than the binary adding machine.

The proof of the theorem is a direct extension of that of Theorem 3.1 in [7] and can be found in Section 2.5 of [6].

2.1. Producing nilpotent state-closed groups. We illustrate in this subsection how certain initial conditions about a simple triple $(G, H, f)$ lead to an understanding of the properties of its state-closed representations, which in turn can be used to construct examples of such triples. Consider the following configuration:

$$
\begin{gathered}
{[G: H]=4,} \\
z \in Z(G), \quad[\langle z\rangle:\langle z\rangle \cap H]=2, \\
f: H \rightarrow G \text { such that } f: z^{2} \rightarrow z .
\end{gathered}
$$

Let $Y=\left\{y_{1}, y_{2}, y_{3}, y_{4}\right\}$ be a right transversal of $H$ in $G$. Then we may choose $y_{1}=e, y_{2}=z$ and $y_{4}=y_{3} z$. We identify $y_{i}$ with its subscript $i$. Thus $z^{\sigma}$ is the permutation $(1,2)(3,4)$ and $z^{\varphi}=\left(e, z^{\varphi}, e, z^{\varphi}\right) .(1,2)(3,4)$; we suppress $\varphi$ from the notation and thus obtain

$$
z=(e, z, e, z) \cdot(1,2)(3,4) .
$$

Let $\mathcal{A}=\operatorname{Aut}(\mathcal{T}(Y)), C=C_{\mathscr{A}}(z)$. We will show that

(i) $C$ is state-closed;

(ii) the commutator equation $[\alpha, \beta]=z$ has the following particular solution in $C$ :

$$
\alpha=(\alpha, \alpha z, \alpha, \alpha)(1,2), \quad \beta=\left(z, z, z^{-1} \beta, z^{-1} \beta\right)(1,3)(2,4) ;
$$

furthermore, the group $R=\langle\alpha, \beta\rangle$ is isomorphic to $F(2,2)$, is recurrent and is finite-state;

(iii) there exists $\kappa \in N_{C}(R)$ defined by

$$
\kappa=\left(\alpha^{3} \kappa^{2}, \alpha^{3} \kappa^{2}, \alpha \kappa^{2}, \alpha \kappa^{2}\right)
$$

such that the group $S=\langle\alpha, \beta, \kappa\rangle$ is a $\mathfrak{\Upsilon}_{3}$ group and has the presentation

$$
\left\{\alpha, \beta, \kappa \mid[\alpha, \beta, \alpha]=[\alpha, \beta, \beta]=[\alpha, \beta, \kappa]=[\alpha, \kappa]=e,[\beta, \kappa]=\alpha^{-2}\right\} .
$$

Proof. It is straightforward to check that the elements of the centralizer $C=C_{A}(z)$ in $\mathcal{A}$ are of eight types:

$$
\begin{array}{ll}
x_{1}=\left(h_{1}, h_{1}, h_{2}, h_{2}\right), & x_{2}=\left(h_{1}, h_{1} z, h_{2}, h_{2}\right)(1,2), \\
x_{3}=\left(h_{1}, h_{1}, h_{2}, h_{2} z\right)(3,4), & x_{4}=\left(h_{1}, h_{1} z, h_{2}, h_{2} z\right)(1,2)(3,4), \\
x_{5}=\left(h_{1}, h_{1}, h_{2}, h_{2}\right)(1,3)(2,4), & x_{6}=\left(h_{1}, h_{1} z, h_{2}, h_{2} z\right)(1,4)(2,3), \\
x_{7}=\left(h_{1}, h_{1}, h_{2}, h_{2} z\right)(1,3,2,4), & x_{8}=\left(h_{1}, h_{1} z, h_{2}, h_{2}\right)(1,4,2,3),
\end{array}
$$


where in each case $h_{1}, h_{2} \in C$. Therefore $C$ is also state-closed.

If $x, x^{\prime} \in C$ are such that $\left[x, x^{\prime}\right]=z$ then there exist $x_{2}, x_{5} \in\left\langle x, x^{\prime}\right\rangle$ such that $\left[x_{2}, x_{5}\right]=z$. Let

$$
x_{2}=\left(h_{1}, h_{1} z, h_{2}, h_{2}\right)(1,2), \quad x_{5}=\left(k_{1}, k_{1}, k_{2}, k_{2}\right)(1,3)(2,4) .
$$

Then $\left[x_{2}, x_{5}\right]=z$ if and only if

$$
h_{2}=k_{1}^{-1} h_{1} k_{1}, \quad\left[h_{1}, k_{1} k_{2}\right]=z .
$$

One solution is $h_{1}=h_{2}=x_{2}, k_{1}=z, k_{2}=z^{-1} x_{5}$. With these choices, rename $x_{2}$ as $\alpha$ and $x_{5}$ as $\beta$; thus

$$
\alpha=(\alpha, \alpha z, \alpha, \alpha)(1,2), \quad \beta=\left(z, z, z^{-1} \beta, z^{-1} \beta\right)(1,3)(2,4) .
$$

It can be verified directly that $\alpha, \beta$ generate a group $R$ isomorphic to $F(2,2)$, that $R$ is finite-state, and recurrent.

We search in the normalizer of $R$ in $C$ for an element $\kappa$ such that $\langle\alpha, \beta, \kappa\rangle$ is nilpotent of class 3 . Clearly, we may assume $\kappa=\left(\kappa_{1}, \kappa_{1}, \kappa_{2}, \kappa_{2}\right)$. Since $\kappa^{-1} \kappa^{\alpha}$, $\kappa^{-1} \kappa^{\beta}$ stabilize the first level of the tree, there exist integers $i, j, k, l, s, t$ such that

$$
\kappa^{-1} \kappa^{\alpha}=\alpha^{2 i} \beta^{2 j} z^{r}, \quad \kappa^{-1} \kappa^{\beta}=\alpha^{2 l} \beta^{2 k} z^{s}
$$

and thus, modulo $R^{\prime}$, we have

$$
\alpha^{\kappa}=\alpha^{1-2 i} \beta^{-2 j}, \quad \beta^{\kappa}=\alpha^{-2 l} \beta^{1-2 k} .
$$

Since the action of $\kappa$ on $R / R^{\prime}$ is nilpotent, the matrix $\left(\begin{array}{ccc}1-2 i & -2 j \\ -2 l & 1-2 k\end{array}\right)$ has determinant 1 and trace 2 . Thus

$$
k=-i, \quad j l=-i^{2} .
$$

Rather than describing all possible solutions we try

$$
i=j=k=0, \quad l=1, \quad r=s=0 .
$$

Then

$$
\kappa=\left(\kappa_{1}, \kappa_{1}, \kappa_{2}, \kappa_{2}\right), \quad \kappa^{\alpha}=\kappa, \quad \kappa^{-1} \kappa^{\beta}=\alpha^{2} .
$$

Now we calculate

$$
\begin{aligned}
\kappa^{-1} \kappa^{\alpha} & =\left(\kappa_{1}^{-1} \kappa_{1}^{\alpha}, \kappa_{1}^{-1} \kappa_{1}^{\alpha}, \kappa_{2}^{-1} \kappa_{2}^{\alpha}, \kappa_{2}^{-1} \kappa_{2}^{\alpha}\right), \\
\kappa^{-1} \kappa^{\beta} & =\left(\kappa_{1}^{-1} \kappa_{2}^{\beta}, \kappa_{1}^{-1} \kappa_{2}^{\beta}, \kappa_{2}^{-1} \kappa_{1}, \kappa_{2}^{-1} \kappa_{1}\right), \\
\alpha^{2} & =\left(\alpha^{2} z, \alpha^{2} z, \alpha^{2}, \alpha^{2}\right) .
\end{aligned}
$$


Thus

$$
\begin{gathered}
\kappa_{1}^{-1} \kappa_{1}^{\alpha}=\kappa_{2}^{-1} \kappa_{2}^{\alpha}=e, \\
\kappa_{1}^{-1} \kappa_{2}^{\beta}=\alpha^{2} z, \quad \kappa_{2}^{-1} \kappa_{1}=\alpha^{2}, \\
\kappa_{1}=\kappa_{2} \alpha^{2}, \quad \kappa_{2}^{-1} \kappa_{2}^{\beta}=\alpha^{4} z
\end{gathered}
$$

and we find that $\kappa_{2}=\alpha \kappa^{2}$ is a solution. Thus

$$
\kappa=\left(\alpha^{3} \kappa^{2}, \alpha^{3} \kappa^{2}, \alpha \kappa^{2}, \alpha \kappa^{2}\right)
$$

satisfies all our conditions.

The group $S=\langle\alpha, \beta, \kappa\rangle$ is a $\mathfrak{\Upsilon}_{3}$ group with the presentation

$$
\left\{\alpha, \beta, \kappa \mid[\alpha, \beta, \alpha]=[\alpha, \beta, \beta]=[\alpha, \beta, \kappa]=[\alpha, \kappa]=e,[\beta, \kappa]=\alpha^{-2}\right\} \text {. }
$$

The groups $R, S$ are the first two terms of an infinite sequence of nilpotent subgroups of $C_{\mathcal{A}}(z)$, which we will construct in Section 5.4.

2.2. State-closed abelian groups. Given $\alpha \in \mathcal{A}$ we indicate the diagonal automorphism $(\alpha, \alpha, \ldots, \alpha)$ by $\alpha^{(1)}$ and inductively $\left(\alpha^{(i)}, \alpha^{(i)}, \ldots, \alpha^{(i)}\right)$ by $\alpha^{(i+1)}$.

The following theorem shows that recurrent abelian groups (no conditions on type) are in a sense small.

Theorem 7. Let $Y=\{1,2, \ldots, m\}, \mathcal{A}=\operatorname{Aut}(\mathcal{T}(Y))$.

(i) Let $G$ be an abelian recurrent subgroup of $\mathcal{A}$ and $C_{\mathcal{A}}(G)$ be the centralizer of $G$ in $\mathcal{A}$. Let $\widehat{G}$ the topological closure of $G$ in $\mathcal{A}$. Then $C_{\mathcal{A}}(G)=\widehat{G}$.

(ii) Let $m$ be a prime number and $G$ an infinite abelian state-closed subgroup of $\mathcal{A}$ which acts transitively on the first level of the tree. Then $C_{\mathcal{A}}(G)=\widehat{G}$.

Proof. (i) Let the vertices of $\mathcal{T}(Y)$ be indexed by sequences from $Y=\{1,2, \ldots, m\}$. Let $G$ induce the permutation group $P$ on the set $Y$. Then $P$ is an abelian transitive permutation group of degree $m$ and is therefore regular; it follows that the stabilizer in $G$ of any $y \in Y$ is the same as the stabilizer of the first level of the tree $H=\operatorname{stab}_{G}(1)$. Since the representation of $G$ is recurrent, the projection $\pi_{v}$ of $\operatorname{stab}_{G}(k)$ on any of its coordinates $v$ produces the group $G$.

For every $\sigma \in P$, choose $a_{\mathbf{0}}(\sigma)=\left(a_{\mathbf{0}}(\sigma)_{1}, \ldots, a_{\mathbf{0}}(\sigma)_{m}\right) \sigma \in G$ which induces $\sigma$ on $Y$. Let $h=\left(h_{1}, h_{2}, \ldots, h_{m}\right) \in H$. Then

$$
h^{a_{\mathbf{0}}(\sigma)}=\left(\left(h_{1}\right)^{a_{\mathbf{0}}(\sigma)_{1}},\left(h_{2}\right)^{a_{\mathbf{0}}(\sigma)_{2}}, \ldots,\left(h_{m}\right)^{a_{\mathbf{0}}(\sigma)_{m}}\right)^{\sigma}=\left(h_{1}, h_{2}, \ldots, h_{m}\right)^{\sigma}
$$

since $h_{i}, a_{\mathbf{0}}(\sigma)_{i} \in G$ which is abelian. On varying $\sigma \in P$ we find that $h=$ $\left(h_{1}, h_{1}, \ldots, h_{1}\right)$. 
Now for every $\sigma \in P$, there exists $a_{\mathbf{1}}(\sigma)=\left(a_{\mathbf{0}}(\sigma), \ldots, a_{\mathbf{0}}(\sigma)\right) \in H$, which induces $\sigma^{(1)}$ modulo $\operatorname{stab}_{\mathcal{A}}(2)$. Thus we produce a sequence $a_{i}(\sigma) \in \operatorname{stab}_{G}(i)$ of elements in $G$ such that $a_{i}(\sigma)=\sigma^{(i)}$ modulo $\operatorname{stab}_{\mathcal{A}}(i+1)$.

Let $\gamma \in C=C_{\mathcal{A}}(G)$. Then

$$
\begin{aligned}
\gamma & =\left(\gamma_{1}, \ldots, \gamma_{m}\right) \sigma \\
\gamma^{\prime} & =\gamma \cdot a_{\mathbf{0}}(\sigma)^{-1}=\left(\gamma_{1}^{\prime}, \ldots, \gamma_{m}^{\prime}\right) \in \operatorname{stab}_{C}(1)
\end{aligned}
$$

and $\gamma_{1}^{\prime}=\cdots=\gamma_{m}^{\prime}$; say $\gamma_{1}^{\prime}$ induces a permutation $\sigma^{\prime}$ on $Y$. Thus

$$
\gamma \cdot a_{\mathbf{0}}(\sigma)^{-1} \cdot a_{\mathbf{1}}\left(\sigma^{\prime}\right)^{-1} \in \operatorname{stab}_{C}(2) .
$$

We produce in this manner a sequence

$$
a_{\mathbf{0}}(\sigma), a_{\mathbf{1}}\left(\sigma^{\prime}\right), a_{\mathbf{2}}\left(\sigma^{\prime \prime}\right), \ldots
$$

of elements of $G$ such that $\gamma$ is equal to the infinite product

$$
a_{\mathbf{0}}(\sigma) a_{\mathbf{1}}\left(\sigma^{\prime}\right) a_{\mathbf{2}}\left(\sigma^{\prime \prime}\right) \ldots
$$

Hence, $C_{\mathcal{A}}(G)=\widehat{G}$.

(ii) Let $m=p$, a prime number. The permutation group $P$ induced on $Y=$ $\{1, \ldots, p\}$ is cyclic, say generated by $\sigma$. Since $G$ is infinite, there exists an $h=$ $\left(h_{1}, h_{1}, \ldots, h_{1}\right) \in H$ such that $h_{1} \notin H$ and therefore we may assume $h_{1}$ induces $\sigma$ on $Y$. We produce elements $a_{i} \in G$ such that $a_{i}=\sigma^{(i)}$ modulo $\operatorname{stab}_{\mathcal{A}}(i+1)$ and the proof continues as previously.

The group $G$ in part (ii) need not be recurrent. For example, let $Y=\{1,2\}$ and $G$ be the cyclic subgroup of $\operatorname{Aut}(\mathcal{T}(Y))$ generated by $\alpha=\left(e, \alpha^{3}\right) \sigma$ where $\sigma$ is the transposition $(1,2)$.

Proposition 1. Let $G$ be a finitely generated abelian group, $H$ a normal subgroup of $G$ such that $\frac{G}{H}$ is cyclic of prime power order. Suppose $(G, H, f)$ is a simple triple. Then either $G$ is finite or free abelian.

Proof. Let $\operatorname{Tor}(G)$ be the torsion subgroup of $G$. Then we have the decompositions

$$
G=\operatorname{Tor}(G) \oplus K, \quad H=\operatorname{Tor}(H) \oplus M
$$

where $\operatorname{Tor}(H) \leq \operatorname{Tor}(G)$ and we may assume that $M \leq K$. Suppose $G$ is a mixed group. Then the first possibility is that $K=M$ and $\frac{\operatorname{Tor}(G)}{\operatorname{Tor}(H)}$ is cyclic. Let $n$ be the exponent of $\operatorname{Tor}(G)$. Then we have $\left(M^{n}\right)^{f}=\left(M^{f}\right)^{n} \leq G^{n}=M^{n}$, a contradiction. The other possibility is that $\operatorname{Tor}(G)=\operatorname{Tor}(H)$ and $\frac{K}{M}$ is finite cyclic; but as $\operatorname{Tor}(H)$ is $f$-invariant, $\operatorname{Tor}(G)=\operatorname{Tor}(H)=\{e\}$, and again we have a contradiction. 


\section{Sub-triples and quotient triples}

A subgroup $K$ of $G$ is called semi-invariant under the action of $f$ provided that $(K \cap H)^{f} \leq K$. If $K \leq H$ and $K^{f} \leq K$ then $K$ is $f$-invariant. Given a triple $(G, H, f)$ and $G_{1} \leq G, H_{1} \leq H \cap G_{1}$ such that $\left(H_{1}\right)^{f} \leq G_{1}$, we call $\left(G_{1}, H_{1},\left.f\right|_{H_{1}}\right)$ a sub-triple. If $N$ is a normal semi-invariant subgroup of $G$ then $\bar{f}: \frac{H N}{N} \rightarrow \frac{G}{N}$ given by $\bar{f}: N h a \rightarrow N h^{f}$ is well defined and $\left(\frac{G}{N}, \frac{H N}{N}, \bar{f}\right)$ is a quotient triple.

Given a triple $(G, H, f)$, we produce a sequence of subtriples $\left(G(i), H(i), f_{i}\right)$ defined as follows:

$$
G(0)=G, \quad H(0)=H, \quad f_{0}=f
$$

and for $i \geq 1$

$$
G(i)=H(i-1)^{f}, \quad H(i)=H(i-1) \cap G(i), \quad f_{i}=\left.f_{i-1}\right|_{H(i)} .
$$

Clearly, if $f$ is an epimorphism, then the sequence stops at $i=0$.

Example 1. The following group $G$ of automorphisms of the binary tree provides an example for which the sequence $G(i)$ is infinite,

$$
G=\left\langle\alpha=\left(1, \alpha \beta^{2}\right) \sigma, \beta=(\alpha, \alpha)\right\rangle .
$$

It is straightforward to check that $G$ is free abelian of rank 2, the subgroup $H=\left\langle\alpha^{2}, \beta\right\rangle$ is of index 2 and the projection of $H$ on the second coordinate is an extension of

$$
f: \alpha^{2} \rightarrow \alpha \beta^{2}, \quad \beta \rightarrow \alpha .
$$

We claim that for $i \geq 1$

$$
G(i)=\left\langle\alpha^{2^{i-1}}, \alpha^{r_{i}} \beta^{2}\right\rangle
$$

where

$$
r_{1}=r_{2}=1 \text { and } r_{i}=1+4 t_{i} \text { such that } t_{i} r_{i-1} \equiv 1\left(\bmod 2^{i-2}\right) \text { for } i \geq 3 .
$$

The assertion is true for $i=1,2$ :

$$
\begin{aligned}
& G(0)=G=\langle\alpha, \beta\rangle, H(0)=H=\left\langle\alpha^{2}, \beta\right\rangle ; \\
& G(1)=H(0)^{f}=\left\langle\alpha \beta^{2}, \alpha\right\rangle=\left\langle\alpha, \beta^{2}\right\rangle, \\
& H(1)=\left\langle\alpha^{2}, \beta^{2}\right\rangle ; \\
& G(2)=H(1)^{f}=\left\langle\alpha \beta^{2}, \alpha^{2}\right\rangle=\left\langle\alpha^{2}, \alpha \beta^{2}\right\rangle .
\end{aligned}
$$

Now suppose

$$
G(i)=\left\langle\alpha^{2^{i-1}}, \alpha^{r_{i}} \beta^{2}\right\rangle
$$


Then

$$
\begin{aligned}
H(i) & =\left\langle\left(\alpha^{r_{i}} \beta^{2}\right)^{2}, \alpha^{2^{i-1}}\right\rangle, \\
G(i+1) & =\left\langle\left(\alpha \beta^{2}\right)^{r_{i}} \alpha^{4},\left(\alpha \beta^{2}\right)^{2^{i-2}}\right\rangle .
\end{aligned}
$$

Viewing $G$ as an additive group with basis $\alpha, \beta$, the generators of $G(i+1)$ are the rows of the matrix $M=\left(\begin{array}{cc}4+r_{i} & 2 r_{i} \\ 2^{i-2} & 2^{i-1}\end{array}\right)$.

Let $m, k$ be integers such that $m r_{i}+k 2^{i-2}=1$ and let $S=\left(\begin{array}{cc}2^{i-2} & -r_{i} \\ m & k\end{array}\right)$. Then $\operatorname{det}(S)=1$ and

$$
S M=\left(\begin{array}{cc}
2^{i} & 0 \\
m\left(4+r_{i}\right)+k 2^{i-2} & 2
\end{array}\right) .
$$

Since $m\left(4+r_{i}\right)+k 2^{i-2}=4 m+m r_{i}+k 2^{i-2}=4 m+1$, we have

$$
G(i+1)=\left\langle\alpha^{2^{i}}, \alpha^{1+4 m} \beta^{2}\right\rangle .
$$

Clearly, $[G: G(i)]=2^{i}$ and therefore $G(i) \neq G(j)$ for $i<j$.

Proposition 2. Let $G$ be group, $H$ a subgroup $G$ and suppose that $(G, H, f)$ is a simple triple such that $G=Z(G) H^{f} H$. Define

$$
G(1)=H^{f}, \quad H(1)=H \cap H^{f}, \quad f_{1}=\left.f\right|_{H(1)} .
$$

Then $\left(G(1), H(1), f_{1}\right)$ is a simple triple.

Proof. Let $Y$ be a right transversal of $H$ in $G$ such that $Y$ is contained in $Z(G) H^{f}$. Let $K$ be a subgroup of $H(1)$, normal in $G(1)$, with $K^{f} \leq K$. Then $K \leq K^{f^{-1}}$ and $K^{f^{-1}}$ is normal in $H$. Thus

$$
K=K^{y} \leq\left(K^{f^{-1}}\right)^{y}=\left(K^{f^{-1}}\right)^{h y}
$$

for all $y \in Y$ and all $h \in H$; that is, $K \leq\left(K^{f^{-1}}\right)^{g}$ for all $g \in G$. Let $M=$ $\bigcap_{g \in G}\left(K^{f^{-1}}\right)^{g}$. Then $M \leq K^{f^{-1}} \leq H$, and $M$ is normal in $G$ such that

$$
K \leq M \leq K^{f^{-1}}, \quad M^{f} \leq K \leq M ;
$$

hence $M=\{e\}=K$.

The above result is a generalization of Lemma 3.2 in [7].

Lemma 1. Let $(G, H, f)$ be a triple. Suppose $B$ is semi-invariant and $\sqrt[G]{B}$ is a group. Then $\sqrt[G]{B}$ is semi-invariant.

Proof. Let $x \in \sqrt[H]{B}$. Then $x \in H$ and $x^{n} \in B \cap H$ for some $n$. Therefore,

$$
\left(x^{n}\right)^{f}=\left(x^{f}\right)^{n} \in(B \cap H)^{f}
$$

and so $x^{f} \in \sqrt[G]{(B \cap H)^{f}} \leq \sqrt[G]{B}$. 
3.1. Facts about nilpotent groups. We list below some facts about nilpotent groups which are either well known (see [5],[8]) or have direct proofs. Let

$$
Z_{i}(G) \quad \text { and } \quad \gamma_{i}(G) \quad(1 \leq i \leq c)
$$

denote the upper and lower central series of $G$, respectively.

I. Let $G$ be a nilpotent group of class $c$.

1. For all $1 \leq i, j \leq c$ :

$$
\begin{aligned}
{\left[\gamma_{i}(G), \gamma_{j}(G)\right] } & \leq \gamma_{i+j}(G), \\
{\left[Z_{i}(G), \gamma_{j}(G)\right] } & \leq Z_{i-j}(G) .
\end{aligned}
$$

2. If $G=Z_{i}(G) H$ for some $i$, then for all $1 \leq j \leq c$ :

$$
\begin{aligned}
\gamma_{j}(G) & \leq Z_{i-j+1}(G) \gamma_{j}(H), \\
\gamma_{i+1}(H) & =\gamma_{i+1}(G) .
\end{aligned}
$$

3. The subset $\operatorname{Tor}(G)$ of $G$ of elements of finite order is a subgroup of $G$.

4. If $Z(G) \leq \operatorname{Tor}(G)$ then $G=\operatorname{Tor}(G)$.

5. Suppose that $N$ is a normal torsion-free subgroup of $G$. Let $x \in G, y \in N$ and $n$ a positive integer. Then

$$
\left[x^{n}, y\right]=e \Longrightarrow[x, y]=e .
$$

6. Suppose that $\operatorname{Tor}(G)$ has finite exponent $s$. If $G=\operatorname{Tor}(G) K$ for some $K \leq G$ then $G^{s}=K^{s}$.

II. Let $G$ be torsion-free nilpotent.

1. Let $K$ be a subgroup of $G$. Then the isolator of $K$ in $G$,

$$
\sqrt[G]{K}=\left\{x \in G \mid x^{n} \in K \text { for some positive integer } n\right\},
$$

is a subgroup of $G$. If furthermore $G$ is finitely generated then $[\sqrt[G]{K}: K]$ is finite.

2. Let $H$ be a subgroup of finite index $m$ in $G$. Then $H \cap Z_{i}(G)=Z_{i}(H)$ for all $i$. Also, $\left[Z_{i}(G): Z_{i}(H)\right]=q_{i}$ is finite for all $i$ and $q_{i}$ divides $q_{j}$ for $i \leq j$.

III. Let $G$ be finitely generated nilpotent group of class $c$.

1. Then $G$ is Hopfian and has a finite Hirsch length denoted by $h(G)$. Also, a subgroup $H$ has finite index in $G$ if and only if $h(H)=h(G)$.

2. Let $H$ be subgroup of finite index in $G$. Then $Z(G)$ and $Z(H)$ have the same Hirsch length. Moreover, $\left[\gamma_{i}(G): \gamma_{i}(H)\right]$ is finite. 


\subsection{Triples for nilpotent groups}

Lemma 2. Let $G$ be a nilpotent group, $(G, H, f)$ a triple, $\bar{G}=\frac{G}{\operatorname{Tor}(G)}$ and $\bar{H}=$ $\frac{H \operatorname{Tor}(G)}{\operatorname{Tor}(G)}$. Then $(\operatorname{Tor}(G), \operatorname{Tor}(H), f),(\bar{G}, \bar{H}, \bar{f})$ are triples. Furthermore, if $G$ is finitely generated and $(G, H, f)$ is simple then $(\bar{G}, \bar{H}, \bar{f})$ is simple.

Proof. The first assertion follows from $\operatorname{Tor}(G) \cap H=\operatorname{Tor}(H)$. Let $\frac{L}{\operatorname{Tor}(G)} \leq \bar{H}$ be the $\bar{f}$-core $(\bar{H})$. Then

$$
L=\operatorname{Tor}(G)(L \cap H)=\operatorname{Tor}(L)(L \cap H)
$$

and $(L \cap H)^{f} \leq L$. By Subsection 3.1, item I.6, there exists $s \geq 1$ such that $L^{s}=(L \cap H)^{s}$. Therefore, $L^{s}$ is $f$-invariant. As $f$ is simple, we have $L^{s}=\{e\}$, $L=\operatorname{Tor}(G)$.

Lemma 3. Let $G$ be a ₹-group, let $H$ be a subgroup of $G$ of finite index in $G$ and let $f: H \rightarrow G$ be a monomorphism. Then $H^{f}$ has finite index in $G$. If $U$ is an $f$-invariant normal subgroup of $H$ then $U \cap Z(H)$ is an $f$-invariant normal subgroup of $G$. If $(G, H, f)$ is simple then $\left(\left\langle H, H^{f}\right\rangle, H, f\right)$ is simple.

Proof. The groups $H$ and $G$ have equal Hirsch lengths, by Subsection 3.1, item I.4. Since $H^{f} \cong H$, it has the same Hirsch length as $G$ and so $H^{f}$ has finite index in $G$. Therefore, $Z(H), Z(H)^{f} \leq Z(G)$. Let $W=U \cap Z(H)$. Then

$$
W^{f} \leq U^{f} \cap Z(H)^{f} \leq U \cap Z(G)=W .
$$

The last assertion follows directly.

Proposition 3. Let $G$ be a $\mathfrak{\mathfrak { T }}_{c}$-group, let $H$ be a subgroup of finite index in $G$, let $f \in \operatorname{Hom}(H, G)$ be a simple monomorphism and let $L \leq G$ be defined by $\frac{L}{Z(G)}=\bar{f}$-core $\left(\frac{H Z(G)}{Z(G)}\right)$. Then the following holds:

(i) $\bar{f}: \frac{H Z(G)}{Z(G)} \rightarrow \frac{G}{Z(G)}$ induced by $f$ is a monomorphism.

(ii) $L, \sqrt[G]{L}$ are abelian, semi-invariant and the corresponding quotient triples $\left(\frac{G}{L}, \frac{H L}{L}, \bar{f}\right),\left(\frac{G}{\sqrt[G]{L}}, \frac{H \sqrt[G]{L}}{\sqrt[G]{L}}, \bar{f}\right)$ are simple.

(iii) If $H L=H \sqrt[G]{L}$ then $L=\sqrt[G]{L}$.

(iv) If $G=H \sqrt[G]{L}$ then $G$ is abelian.

Proof. Let $h \in H$ such that $Z(G) h \in \operatorname{ker}(\bar{f})$. Then $h^{f} \in Z(G)$. As $Z(H)^{f}=$ $Z\left(H^{f}\right) \leq Z(G)$ and $\operatorname{ker}(f)=\{e\}$, it follows that $h \in Z(H) \leq Z(G)$. 
We have $Z(G) \leq L \leq Z(G) H$. Let $M=L \cap H$. Then $M$ is a normal subgroup of $H, L=Z(G)+M$ and $L^{\prime}=M^{\prime}$. Also $(Z(G) x)^{\bar{f}}=Z(G) x^{f} \in Z(G) M$ for all $x \in M$; that is, $M^{f} \leq Z(G) M$. Therefore $\left(M^{\prime}\right)^{f} \leq M^{\prime}$. Since $f$ is simple, $M$ and $L$ are abelian and so $\sqrt[G]{L}$ is abelian.

Write $\bar{G}=\frac{G}{Z(G)}$ and $\bar{L}=\frac{L}{Z(G)}$. Since $\sqrt[G]{L}$ is abelian, it follows easily that $\sqrt[\bar{G}]{\bar{L}}=\frac{\sqrt[G]{L}}{Z(G)}$. If $x \in \sqrt[H]{L}$ then there exists $n$ such that $x^{n} \in L \cap H=M$; therefore $\left(x^{n}\right)^{f}=\left(x^{f}\right)^{n} \in L$ and $x^{f} \in \sqrt[G]{L}$. The assertion that $\left(\frac{G}{\sqrt[G]{L}}, \frac{H \sqrt[G]{L}}{\sqrt[G]{L}}, \bar{f}\right)$ is simple is now clear.

Let $\bar{G}=\frac{G}{L}$ and $\bar{H}=\frac{H L}{L}$. Then $\frac{\sqrt[G]{L}}{L}=\operatorname{Tor}(\bar{G})$ and it follows that from Lemma 2 that $\left(\frac{G}{\sqrt[G]{L}}, \frac{H^{G} \sqrt{L}}{\sqrt[G]{L}}, \bar{f}\right)$ is simple. Suppose that $H L=H \sqrt[G]{L}$. Then $\bar{H}=\bar{H} \operatorname{Tor}(\bar{G})$ and the equalities $\operatorname{Tor}(\bar{H})=\operatorname{Tor}(\bar{G})=\{L\}$ follow; that is, $L=\sqrt[G]{L}$.

Suppose $G=\sqrt[G]{L} H$. Then working modulo $L$, we have $\sqrt[G]{L}=T(G)$ and so there exists $s \geq 1$ such that $G^{s}=H^{s}$. In other words, going back to $G$, we have $G^{s} L=H^{s} L$. It follows from $L=Z(G) M$ that

$$
\left[L, G^{s}\right]=\left[L, H^{s}\right]=\left[M, G^{s}\right]=\left[M, H^{s}\right] ;
$$

hence $\left[M, H^{s}\right]$ is an $f$-invariant subgroup. Therefore,

$$
\left[M, H^{s}\right]=\left[M, G^{s}\right]=\{e\}, \quad M \leq Z(G),
$$

and

$$
L=Z(G)=\sqrt[G]{L}, \quad G=Z(G) H, \quad G^{\prime}=H^{\prime}=\{e\} .
$$

We show in the next example that $\sqrt[G]{L}$ can be different from $L$.

Example 2. Let $G=F(2,2)$ freely generated by $x_{1}, x_{2}$. Let $n>1$ and let $H=$ $\left\langle x_{1}^{n}, x_{2}^{n}\right\rangle$. Then $Z(H)=\left\langle\left[x_{2}, x_{1}\right]^{n^{2}}\right\rangle$. The map $f: x_{1}^{n} \rightarrow x_{1}^{n}, x_{2}^{n} \rightarrow x_{2}$ extends to a monomorphism from $H$ into $G$ where $f:\left[x_{2}, x_{1}\right]^{n^{2}} \rightarrow\left[x_{2}, x_{1}\right]^{n}$. It is then clear that $f$ is simple, $L=Z(G)\left\langle x_{1}^{n}\right\rangle$ and $\sqrt[G]{L}=Z(G)\left\langle x_{1}\right\rangle$.

\section{Kernel versus core}

Proposition 4. Let $K, P$ be groups, $P$ a transitive permutation group on the set $Y=\{1, \ldots, m\}$ and $P_{1}$ the stabilizer of 1 in $P$. Furthermore, let $W$ be the wreath product $K \operatorname{wr}_{Y} P$ and let $W$ act on $Y$ as $P$. Let $B=K^{Y}$ and $W_{1}=B P_{1}$. Consider a nilpotent subgroup $G$ of $W$ which induces a transitive group on $Y$. Let $x=$ $\left(x_{1}, x_{2}, \ldots, x_{m}\right) \sigma \in G_{1}=G \cap W_{1}$. If $x_{1}$ has finite order then $x$ also has finite order.

Proof. Suppose that there exists an $x=\left(x_{1}, x_{2}, \ldots, x_{m}\right) \sigma$ where $\sigma \in P_{1}$ such that $x_{1}$ has finite order, yet $o(x)$ is infinite. Then $x^{\prime}=\left(x^{o\left(x_{1}\right)}\right)^{o(\sigma)} \in B_{G}=G \cap B$ 
and $x^{\prime}=\left(e, x_{2}^{\prime}, \ldots, x_{m}^{\prime}\right)$ has infinite order. Let $X$ be the set of non-trivial $x \in B_{G}$ such that each $x_{i}=e$ or $o\left(x_{i}\right)$ is infinite. Choose $x \in X$ such that first, $x$ has a maximum number of trivial entries and second, $x \in \gamma_{j}(G)$ for a maximum $j$. Let $g=\left(g_{1}, g_{2}, \ldots, g_{m}\right) \rho \in G$ where $\rho \in P$ and $o(\rho)=r$. Then $\left[x, g^{r}\right] \in B_{G}$ has at least the same number of trivial entries as $x$ and $\left[x, g^{r}\right] \in \gamma_{j+1}(G)$. Therefore $\left[x, g^{r}\right]$ has finite order. Hence in the torsion-free nilpotent group $\bar{G}=\frac{G}{\operatorname{Tor}(G)}$, we have $\left[\bar{x}, \overline{g^{r}}\right]=\bar{e}$ and so $[\bar{x}, \bar{g}]=\bar{e}$; that is, $[x, g]$ has finite order. We may assume that $x_{1}=e$ and let $i$ be such $x_{i}$ has infinite order. Now let $g=\left(g_{1}, g_{2}, \ldots, g_{m}\right) \rho \in G$ be such that $(i) \rho=1$. Then

$$
[x, g]=x^{-1} x^{g}=\left(e, x_{2}^{-1}, \ldots, x_{m}^{-1}\right)\left(e, x_{2}^{g_{2}}, \ldots, x_{m}^{g_{m}}\right)^{\rho}=\left(x_{i}^{g_{i}}, *, \ldots, *\right),
$$

which has infinite order; a contradiction is reached.

Theorem 8. Let $G$ be a nilpotent group, $H$ a subgroup of finite index $m$ in $G$, $f \in \operatorname{Hom}(H, G)$ and $L=f$-core $(H)$. Then

(i) $\operatorname{ker}(f) \leq \sqrt[H]{L}$;

(ii) $\operatorname{Tor}(H)^{f} \leq \operatorname{Tor}\left(H^{f}\right) \leq(\sqrt[H]{L})^{f}$;

(iii) if $L=\{e\}$ then $\operatorname{Tor}(H)^{f}=\operatorname{Tor}\left(H^{f}\right)$;

(iv) if $G$ is finitely generated and $f$ an epimorphism, then $\sqrt[G]{L}=L$.

Proof. The triple $(G, H, f)$ provides us with a state-closed representation $\varphi: \frac{G}{L} \rightarrow \operatorname{Aut}(\mathcal{T}(Y))$ for $Y=\{1, \ldots, m\}$ and where for $h \in H$ we have

$$
h^{\varphi}=\left(h^{f \varphi}, *, \ldots, *\right) \sigma
$$

and $(1)^{\sigma}=1$.

(i) If $h \in \operatorname{ker}(f)$ then $h^{\varphi}=(e, *, \ldots, *) \sigma$, and by the previous proposition $h^{\varphi}$ has finite order. As $L=\operatorname{ker}(\varphi)$, we have $h \in \sqrt[H]{L}$ and we are done.

(ii) The first inclusion is clear. Now suppose $x \in \operatorname{Tor}\left(H^{f}\right)$; that is, $x=h^{f}$ and $x^{n}=e$ for some $n$. Then

$$
e=\left(h^{f}\right)^{n}=\left(h^{n}\right)^{f}, \quad h^{n} \in \operatorname{ker}(f), \quad h \in \sqrt[H]{\operatorname{ker}(f)} \leq \sqrt[H]{L}, \quad x \in(\sqrt[H]{L})^{f} .
$$

If $L=\{e\}$ then $\sqrt[H]{L}=\operatorname{Tor}(H)$ and the result follows from $\operatorname{Tor}(H)^{f} \leq \operatorname{Tor}\left(H^{f}\right) \leq$ $\operatorname{Tor}(H)^{f}$.

(iii) follows immediately from (ii).

(iv) Since $G$ is finitely generated, $\operatorname{Tor}(G)$ is a finite group. Suppose initially that $L$ is trivial. Then $\sqrt[G]{L}=\operatorname{Tor}(G)$ and by item (ii), $\operatorname{Tor}(H)^{f} \leq \operatorname{Tor}(G) \leq \operatorname{Tor}(H)^{f}$; thus $\operatorname{Tor}(H)^{f}=\operatorname{Tor}(G)$. As $\operatorname{Tor}(H) \leq \operatorname{Tor}(G)$, we have $\operatorname{Tor}(H)=\operatorname{Tor}(G)$ and as $f$ is simple, we conclude that $\operatorname{Tor}(G)$ is trivial.

In the general case we consider the triple $\left(\frac{G}{L}, \frac{H}{L}, \bar{f}\right)$. Then $\bar{f}$ is a simple epimorphism and therefore Tor $\left(\frac{G}{L}\right)=\{L\}$; that is, $\sqrt[G]{L}=L$. 
Corollary 1. Let $G$ be a torsion-free nilpotent group, $H$ a subgroup of finite index $m$ and $f: H \rightarrow G$ a homomorphism. Then

$$
f \text { simple } \Longrightarrow \operatorname{ker}(f)=\{e\} .
$$

Suppose that $\operatorname{ker}(f)=\{e\}$. Then

$$
f \text { simple } \Longleftrightarrow f: Z(H) \rightarrow Z(G) \text { simple. }
$$

Proof. The first assertion is a direct application of part (i) of the theorem. Suppose $G$ is finitely generated; then $Z(H) \leq Z(G)$. It follows easily that $f$ simple implies that $\left.f\right|_{Z(H)}: Z(H) \rightarrow Z(G)$ simple. On the other hand, suppose that $f: Z(H) \rightarrow$ $Z(G)$ is simple and let $K$ be a nontrivial subgroup of $H$, normal in $G$ and $f$-invariant. Then $K \cap Z(H)=\{e\}$ and

$$
\begin{aligned}
(K \cap Z(H))^{f} & \leq K \cap Z(H)^{f}=K \cap Z\left(H^{f}\right) \\
& \leq K \cap Z(G)=K \cap H \cap Z(G)=K \cap Z(H) .
\end{aligned}
$$

Example 3. (1) A simple triple $(G, H, f)$ where $G$ is finite and $\operatorname{ker}(f) \neq\{e\}$.

Let $p$ be a prime number, $Y=\{1,2, \ldots, p\}$ and $\sigma$ the permutation $(1,2, \ldots, p)$. Let $W_{s}$ be the group of automorphisms of the $p$-adic tree $\mathcal{T}(Y)$ generated by

$$
\sigma_{0}=\sigma, \sigma_{1}=\left(e, \ldots, e, \sigma_{0}\right), \ldots, \sigma_{s}=\left(e, \ldots, e, \sigma_{s-1}\right),
$$

The $W_{s}$ is the $s$-iterated wreath product $\left(\left(\left(C_{p}\right.\right.\right.$ wr $\left.\left.\left.\ldots\right) \mathrm{wr}\right) C_{p}\right)$ wr $C_{p}\left(=W_{s-1} \operatorname{wr} C_{p}\right)$. Let $H=\operatorname{stab}_{W_{s}}(1)$ and $\pi_{1}: H \rightarrow W_{s}$. Then $\left[W_{s}: H\right]=p, \pi_{1}(H) \cong W_{s-1}$ and $\operatorname{ker}(f)=\{e\} \times W_{s-1} \times \cdots \times W_{s-1}$.

(2) A simple triple $(G, H, f)$ where $G$ is of mixed type and $\operatorname{ker}(f) \neq\{e\}$.

Let $G=(C$ wr $D)\langle x\rangle$ where $C=\langle c\rangle, D=\langle d\rangle$ each of order $p$, and $x$ of infinite order inducing conjugation by $d$ on $C$ wr $D$. Therefore, $G$ is a nilpotent group with $Z(G)=\left\langle z, d x^{-1}\right\rangle$ where $z=c c^{d} \ldots c^{d^{p-1}}$. Let $H=\left\langle C^{D}, x^{p}\right\rangle=$ $\left\langle c, c^{d}, \ldots, c^{d^{p-2}}, z, x^{p}\right\rangle$ and $M=\langle d, x\rangle$ an abelian group of type $\mathbb{Z}_{p} \times \mathbb{Z}$. Then $H$ is abelian of type $\left(\mathbb{Z}_{p}\right)^{p} \times \mathbb{Z},[G: H]=p^{2}, Z(H)=\left\langle z, x^{p}\right\rangle$. The extension of the map

$$
c \rightarrow 1, c^{d} \rightarrow 1, \ldots, c^{d^{p-2}} \rightarrow 1, z \rightarrow d, x^{p} \rightarrow x
$$

produces an epimorphism $f: H \rightarrow M$. Then $\operatorname{ker}(f)=\left\langle c, c^{d}, \ldots, c^{d^{p-2}}\right\rangle$. Note that the only subgroup of $\operatorname{ker}(f)$ which is normal in $\langle c, d\rangle$ is the trivial subgroup. Let $K$ be an $f$-invariant subgroup of $H$ normal in $G$. Then

$$
K^{f} \leq K \cap M \leq H \cap M=\left\langle x^{p}\right\rangle .
$$

Therefore $K^{f}=\{e\}, K \leq \operatorname{ker}(f)$ and so $K=\{e\}$.

(3) A triple $(G, H, f)$ where $G$ is a $\mathfrak{\Upsilon}$-group and $f$-core $(H)=Z(H)$. 
Let $G=F(2,2)$ freely generated by $a, b$. Let $H$ be the subgroup generated by $a^{3}, b^{2}$. Then $[G: H]=36$. Define the endomorphism $f: H \rightarrow G$ extended from $f: a^{3} \rightarrow a^{2}, b^{2} \rightarrow b^{3}$. Then $f:\left[a^{3}, b^{2}\right] \rightarrow\left[a^{2}, b^{3}\right]$. Thus $f$-core $(H)=$ $\left\langle[a, b]^{6}\right\rangle=Z(H)$.

\section{Simple triples for $\mathfrak{T}$-groups}

\subsection{Nilpotent groups: 2-generated or of class 2}

Lemma 4. Let $G$ be a $\mathfrak{T}_{2}$-group. Then there exists a subgroup $K$ of $G$ such that $G=Z(G) K, K^{\prime}=Z(K)$.

Proof. As $Z(G)$ is isolated in $G$, then $\frac{Z(G)}{G^{\prime}}$ has a complement $\frac{K}{G^{\prime}}$ in $\frac{G}{G^{\prime}}$. Therefore

$$
\begin{aligned}
G & =Z(G) K, G^{\prime}=K^{\prime}, \\
G^{\prime} & =Z(G) \cap K=Z(K) .
\end{aligned}
$$

Theorem 9. Let $G$ be a $\mathfrak{\mathfrak { I }}_{c}$-group with $c \leq 2$ and let $H$ be a subgroup of finite index in $G$. Then there exists a subgroup $K$ of finite index in $H$, which admits a strongly simple epimorphism $f: K \rightarrow G$.

Proof. The case $G$ abelian is obvious; so let $G$ have nilpotency class 2. Choose $\left\{Z(G) a_{1}, \ldots, Z(G) a_{d}\right\}$, a free generating set of $\frac{G}{Z(G)}$, and let $A=\left\langle a_{1}, a_{2}, \ldots, a_{d}\right\rangle$. Then, by the previous lemma, $Z(A)=A^{\prime}$ and $Z(G)=U_{0} \oplus U_{1}$ where $U_{1}=$ $\sqrt[G]{Z(A)}$. It follows that $G=U_{0} \oplus U_{1} A$.

There exists a generating set $\left\{x_{1}, x_{2}, \ldots, x_{d}\right\}$ of $A$ such that modulo $Z(G)$ we have $H=\left\langle x_{1}^{k_{1}}, x_{2}^{k_{2}}, \ldots, x_{d}^{k_{d}}\right\rangle$ where $k_{i} \geq 1$. Thus there exist $c_{i} \in Z(G)$ such that $b_{i}=c_{i} x_{i}^{k_{i}} \in H(1 \leq i \leq d)$. Define $B=\left\langle b_{1}, b_{2}, \ldots, b_{d}\right\rangle$. Then

$$
\begin{aligned}
H & =B Z(H), \\
Z(B) & =B^{\prime}=\left\langle\left[x_{i}, x_{j}\right]^{k_{i} k_{j}} \mid i<j\right\rangle, \\
B^{\prime} & \leq A^{\prime}=\left\langle\left[x_{i}, x_{j}\right] \mid i<j\right\rangle .
\end{aligned}
$$

Let $V_{1}=\sqrt[H]{Z(B)}$. Then $V_{1} \leq U_{1}$ and $\left[U_{1}: V_{1}\right]=r_{1}$ is finite. Now we prove that we may choose $U_{0}$ such that $H=V_{0} \oplus V_{1} B$ where $V_{0} \leq U_{0}$. We argue in $\frac{Z(G)}{V_{1}}$. Since $\frac{U_{1}}{V_{1}}=\operatorname{Tor}\left(\frac{Z(G)}{V_{1}}\right)$, there exists $W_{0} \leq Z(G)$ such that $\frac{Z(G)}{V_{1}}=\frac{W_{0} \oplus V_{1}}{V_{1}} \oplus \frac{U_{1}}{V_{1}}$ and $\frac{Z(H)}{V_{1}} \leq \frac{W_{0} \oplus V_{1}}{V_{1}}$. It follows from $V_{1} \leq Z(H) \leq W_{0} \oplus V_{1}$ that $Z(H)=$ $\left(Z(H) \cap W_{0}\right) \oplus V_{1}$. Let $\left[U_{0}: V_{0}\right]=r_{0}$. 
Now let $r=\operatorname{lcm}\left(r_{0}, r_{1}\right), k=\operatorname{lcm}\left\{k_{i} \mid 1 \leq i \leq d\right\}$. Define the subgroups

$$
B_{0}=\left\langle\left(c_{i} x_{i}^{k_{i}}\right)^{r \frac{k}{k_{i}}} \mid 1 \leq i \leq d\right\rangle \quad \text { and } \quad K=Z(G)^{r^{2} k^{2}} B_{0} .
$$

Then $K$ is a subgroup of finite index in $H$ and

$$
K=U_{0}^{r^{2} k^{2}} \oplus U_{1}^{r^{2} k^{2}} B_{0} .
$$

Now consider the map

$$
\gamma: z \rightarrow z^{r^{2} k^{2}}, \quad x_{i} \rightarrow\left(c_{i} x_{i}^{k_{i}}\right)^{r \frac{k}{k_{i}}} .
$$

We note that if the map $\gamma$ extends to an endomorphism from $G$ onto $K$ then

$$
\begin{gathered}
\gamma: U_{0} \rightarrow U_{0}^{r^{2} k^{2}}, \quad U_{1} \rightarrow U_{1}^{r^{2} k^{2}}, \\
{\left[x_{i}, x_{j}\right] \rightarrow\left[x_{i}, x_{j}\right]^{r^{2} k^{2}} \quad(1 \leq i \leq d) .}
\end{gathered}
$$

To prove that $\gamma$ extends to an endomorphism, it is sufficient to observe that if for some $u_{1} \in U_{1}$ and integer $s$ we have that $u_{1}^{s}$ is a word $w\left(\left[x_{i}, x_{j}\right]\right)$ in the commutators $\left[x_{i}, x_{j}\right]$, then we have in the extension $\gamma: u_{1}^{s} \rightarrow\left(u_{1}^{r^{2} k^{2}}\right)^{s}=\left(u_{1}^{s}\right)^{r^{2} k^{2}}$ on the one hand and $\gamma: w\left(\left[x_{i}, x_{j}\right]\right) \rightarrow w\left(\left[x_{i}, x_{j}\right]^{r^{2} k^{2}}\right)=w\left(\left[x_{i}, x_{j}\right]\right)^{r^{2} k^{2}}$ on the other, and the two images coincide.

Example 4. Given a simple triple $(G, H, f)$ where $G$ is a I-group, a question may be posed as to whether assuming $\left.f\right|_{Z(H)}: Z(H) \rightarrow Z(G)$ is an epimorphism implies that $f$ itself is an epimorphism. The following is a counterexample: let $G=F(2,2)$ freely generated by $a, b$. Write $[a, b]=z$ and let $H_{1}=\left\langle a^{4}, b^{4}, z^{4}\right\rangle$, $H_{2}=\left\langle a^{2}, b^{2}, z\right\rangle$. Then $f: a^{4} \rightarrow a^{2}, b^{4} \rightarrow b^{2}, z^{4} \rightarrow z$ extends to an isomorphism from $H_{1}$ onto $H_{2}$ and $f$ is simple. Therefore $Z\left(H_{1}\right)^{f}=Z(G)$, yet $f$ is not an epimorphism.

Lemma 5. Let $G$ is be a 2-generated $\mathfrak{\Im}_{c}$-group. Then $G^{\prime}=Z_{c-1}(G)$.

Proof. We have $G^{\prime} \leq Z_{c-1}(G)$, and $\frac{G}{G^{\prime}}, \frac{G}{Z_{c-1}(G)}$ are 2-generated non-cyclic abelian groups. Since $\frac{G}{Z_{c-1}(G)}$ is a is torsion-free quotient of $\frac{G}{G^{\prime}}$, the result follows.

Theorem 10. Let $G$ be 2-generated $\mathfrak{T}_{c}$-group. Suppose that $H$ is a proper normal subgroup of $G$ of finite index $m$ which is isomorphic to $G$. Then $G$ is abelian.

Proof. Let $G$ be generated by $a_{1}, a_{2}$ and suppose $c \geq 2$.

First, we will argue the case $c=2$. Then $G$ is isomorphic to $F(2,2)$ and $G^{\prime}=Z(G)=\left\langle\left[a_{1}, a_{2}\right]\right\rangle$. We may choose the generators $a_{1}, a_{2}$ such that $H Z(G)=$ $\left\langle a_{1}^{m_{1}}, a_{2}^{m_{2}}\right\rangle Z(G)$. Thus

$$
H^{\prime}=Z(H)=\left\langle\left[a_{1}, a_{2}\right]^{m_{1} m_{2}}\right\rangle .
$$


We have from $\left[H Z(G), a_{i}\right](i=1,2)$

$$
\begin{gathered}
{\left[a_{1}^{m_{1}}, a_{2}\right]=\left[a_{1}, a_{2}\right]^{m_{1}}, \quad\left[a_{1}, a_{2}^{m_{2}}\right]=\left[a_{1}, a_{2}\right]^{m_{2}},} \\
{\left[a_{1}, a_{2}\right]^{m_{1}},\left[a_{1}, a_{2}\right]^{m_{2}} \in H \cap Z(G)=Z(H) .}
\end{gathered}
$$

Therefore, $m_{1} m_{2}= \pm 1$. Hence $H G^{\prime}=G=H$.

Let $c \geq 2$ and $f: H \rightarrow G$ be an epimorphism. Then $f$ induces an epimorphism $\bar{f}: \frac{H Z_{c-2}(G)}{Z_{c-2}(G)} \rightarrow \frac{G}{Z_{c-2}(G)}$, as $H \cap Z_{c-2}(G)=Z_{c-2}(H)$. The class 2 case leads to $G=H Z_{c-2}(G)$. Therefore

$$
\frac{G}{Z_{c-2}(H)}=\frac{H}{Z_{c-2}(H)} \oplus \frac{Z_{c-2}(G)}{Z_{c-2}(H)}
$$

Also, since $\frac{G}{Z_{c-2}(H)}$ and $\frac{H}{Z_{c-2}(H)}$ are 2-generated, we reach $Z_{c-2}(G)=Z_{c-2}(H)$ and thus, $G=H$; again, a contradiction.

\subsection{Derived length and nilpotency class}

Theorem 11. Let $G$ be a $\mathfrak{\Upsilon}$-group, let $H$ be a subgroup of finite index $m>1$ and let $f: H \rightarrow G$ be simple. Then $s(G) \leq l(m)$.

Proof. We may suppose that $G$ is non-abelian. By Corollary $1, f$ is a monomorphism. As $H^{f}$ is a subgroup of finite index in $G$, we have $Z(H), Z(H)^{f} \leq Z(G)$. Clearly, $Z(G)$ is not contained in $H$; for otherwise $Z(G)=Z(H)$ is $f$-invariant.

Consider the triple $\left(\frac{G}{Z(G)}, \frac{H Z(G)}{Z(G)}, \bar{f}\right)$. Then the index $\left[\frac{G}{Z(G)}: \frac{H Z(G)}{Z(G)}\right]$ is a proper divisor of $m$. Define $L \leq G$ by $\frac{L}{Z(G)}=\bar{f}$-core $\left(\frac{H Z(G)}{Z(G)}\right)$. By Proposition 3, both $L$, $\sqrt[G]{L}$ are abelian and the triples $\left(\frac{G}{L}, \frac{H L}{L}, \bar{f}\right),\left(\frac{G}{\sqrt[G]{L}}, \frac{H \sqrt[G]{L}}{\sqrt[G]{L}}, \bar{f}\right)$ are simple.

Now we consider the chain of subgroups

$$
H \leq H L \leq H \sqrt[G]{L} \leq G
$$

Since $H Z(G) \leq H L$ and $Z(G) \not H$, we have that $H$ is a proper subgroup of $H L$. By Proposition 3, if $H L=H \sqrt[G]{L}$ then $L=\sqrt[G]{L}$ and since $G$ is non-abelian, $H \sqrt[G]{L} \neq G$

We apply induction on $l(m)$. If $L=\sqrt[G]{L}$ then $\frac{G}{L}$ is torsion-free, $\left[\frac{G}{L}: \frac{H L}{L}\right]=m^{\prime}$ and $l\left(m^{\prime}\right)<l(m)$; therefore $s\left(\frac{G}{L}\right) \leq l\left(m^{\prime}\right)$ and since $L$ is abelian, $s(G) \leq l\left(m^{\prime}\right)+1 \leq$ $l(m)$. If $L \neq \sqrt[G]{L}$ then $H L \neq H \sqrt[G]{L}$ and therefore $\left[\frac{G}{\sqrt[G]{L}}: \frac{H^{G} \sqrt{L}}{\sqrt[G]{L}}\right]=m^{\prime \prime}$ $l\left(m^{\prime \prime}\right)<l(m)$, and the argument proceeds as in the previous case.

Corollary 2. Let $G$ be a finitely generated nilpotent group. Suppose that $[G: H]=p$ is a prime number and $(G, H, f)$ a simple triple. Then $G$ is a finite p-group (no restriction on the nilpotency class or derived length) or is free abelian. 
Proof. Proceed by induction on the order of $\operatorname{Tor}(G)$. If $G$ is torsion-free then by the previous theorem, $G$ is abelian.

If $\operatorname{Tor}(G) \neq\{e\}$ then $\operatorname{Tor}(G)$ is not contained in $H$. Since $H$ is a maximal subgroup of $G$, by Proposition $2\left(H^{f}, H \cap H^{f}, f\right)$ is a simple triple of degree $p$. Since $\left|\operatorname{Tor}\left(H^{f}\right)\right|<|\operatorname{Tor}(G)|$ we conclude that $H^{f}$ is finite or torsion-free. In the first case, it follows that $G$ is finite. In the second case, $\operatorname{ker}(f)=\operatorname{Tor}(H)$ is normal in $G$ and $f$-invariant; therefore, $\operatorname{ker}(f)=\{e\}$ and $G$ is torsion-free, contrary to the assumption.

To justify that neither $s(G)$ nor $c(G)$ can be bounded in case $G$ is finite, we recall $W_{s}$, the iterated wreath product of cyclic groups of order $p$ in Example $3(1)$.

Theorem 12. Let $G$ be a $\mathfrak{\Upsilon}$-group, $H$ a subgroup of finite index $m$. Suppose that $f: H \rightarrow G$ is strongly simple. Then $\bar{f}: \frac{H Z(G)}{Z(G)} \rightarrow \frac{G}{Z(G)}$ is also strongly simple. Furthermore, $c(G) \leq l(m)$.

Proof. Let $G$ be non-abelian. Suppose that there exists a nontrivial subgroup $\frac{L}{Z(G)}$ of $\frac{Z(G) H}{Z(G)}$ which is $\bar{f}$-invariant; then there exists $K \leq H$ such that $L=Z(G) K$; thus $L^{\prime}=K^{\prime}$ is $f$-invariant. Since $f$ is strongly simple, $L$ is free abelian of finite rank and we may assume that $L=Z(G) \oplus K$, as $Z(G)$ is isolated. The decomposition of $L$ provides us with uniquely defined homomorphisms

$$
\zeta \in \operatorname{Hom}(K, Z(G)), \quad \gamma \in \operatorname{Hom}(K, K)
$$

defined by $k^{f}=k^{\zeta}+k^{\gamma}$ for all $k \in K$. We note that $\gamma$ is a monomorphism whose characteristic polynomial $\mu$ is non-constant.

Since $[L: Z(H) \oplus K]$ is finite, $f$ extends to an automorphism $\hat{f}$ of $\mathbb{Q} \otimes L$, say having characteristic polynomial $\rho$; clearly, $\mu$ is a factor of $\rho$. However, since $f$ is simple, $\rho$ does not have monic integral polynomial factors of positive degree; a contradiction.

Induction on $c(G)$ leads directly to a proof of the last assertion.

The following natural example shows that the above limit is satisfactory.

Example 5. Let $V_{n}$ be the additive free $\mathbb{Z}$-module of rank $n$ generated by $v_{i}$ $(1 \leq i \leq n)$ and let $x_{n} \in \mathrm{GL}\left(V_{n}\right)$ be defined by

$$
x_{n}: v_{i} \rightarrow v_{i}+v_{i+1}, \quad v_{n} \rightarrow v_{n} \quad(1 \leq i \leq n-1) .
$$

Also, let $G_{n}$ be the semidirect product $G_{n}=V_{n}\left\langle x_{n}\right\rangle$. Then $G_{n}$ is a $\mathfrak{T}_{n}$-group. Let $p$ be a prime number, $W_{n}=p V_{n}, x^{\prime}=v_{n} x$ and $H_{n}=W_{n}\left\langle x_{n}^{\prime}\right\rangle$. Then $\left[G_{n}: H_{n}\right]=p^{n}$ and

$$
f_{n}: p v_{i} \rightarrow v_{i}, \quad x_{n}^{\prime} \rightarrow x_{n} \quad(1 \leq i \leq n)
$$

extends to a strongly simple epimorphism $f: H \rightarrow G$. 
5.3. Triples with degree a product of two primes. Let $(G, H, f)$ be a simple triple of degree $m=p q$, a product of two primes. In contrast to the prime degree, here we have a greater variety of groups. However, we do not know of examples of simple

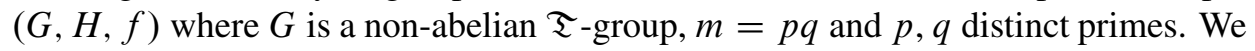
show below that $G$ can be a mixed non-abelian group.

Example 6. (1) A mixed nilpotent group $G$ of class 2, with $[G: H]=p^{2}$.

Let $G=\left\langle a, u, v \mid u^{p}=v^{p}=[u, v]=e, u^{a}=u v, v^{a}=v\right\rangle$ of type $\left(\mathbb{Z}_{p} \times \mathbb{Z}_{p}\right) \mathbb{Z}$. Let $H=\left\langle u, a^{p}\right\rangle$ and $K=\langle v, a\rangle$. Then $[G: H]=p^{2}$ and $H, K$ are abelian of type $\mathbb{Z}_{p} \times \mathbb{Z}$.

Define $f: H \rightarrow K$ by $f: u \rightarrow v, a^{p} \rightarrow a$. Then $f$ is a simple homomorphism; for

$$
u^{a^{p}}=u v^{p}=u, \quad f: u^{a^{p}}(=u) \rightarrow v^{a}(=v) .
$$

(2) A mixed nilpotent group $G$ of class 2, with $[G: H]=p q$ where $p$ is a prime, $q=1+t p$ and $(G, H, f)$ simple.

Let $D=\left\langle a, b \mid a^{p^{2}}=b^{p}=e, a^{b}=a^{1+p}\right\rangle$, a group of order $p^{3}$. Let $G=D .\langle x\rangle$ where $x$ is of infinite order and acts on $D$ as conjugation by $b$. Then

$$
Z(G)=\left\langle a^{p}, b^{-1} x\right\rangle .
$$

We observe that $\left(b^{-1} x\right)^{p}=x^{p}$. Let $q=1+t p$ and let

$$
H=\left\langle a^{p}, b, x^{q}\right\rangle, \quad K=\langle b, x\rangle .
$$

Then $H$ is abelian, has index $p q$ in $G$, has type $\mathbb{Z}_{p} \times \mathbb{Z}_{p} \times \mathbb{Z}$, and $K$ is abelian of type $\mathbb{Z}_{p} \times \mathbb{Z}$. Moreover,

$$
H \cap Z(G)=\left\langle a^{p}, b^{-1} x^{q}\right\rangle
$$

since $b^{-1} x \cdot\left(x^{p}\right)^{t}=b^{-1} x^{q}$. Now the map

$$
f: a^{p} \rightarrow b, \quad b \rightarrow e, x^{q} \rightarrow x
$$

extends to an epimorphism $f: H \rightarrow K$. To prove that $f$ is simple, we observe that

$$
f: H \cap Z(G) \rightarrow\langle b, x\rangle, \quad H \cap\langle b, x\rangle=\left\langle b, x^{q}\right\rangle, \quad f:\left\langle b, x^{q}\right\rangle \rightarrow\langle x\rangle .
$$

Theorem 13. Let $G$ be a $\mathfrak{T}_{c}$-group, $(G, H, f)$ a simple triple of degree $p q$ where $p, q$ are (not necessarily distinct) prime numbers and let $\frac{L}{Z(G)}=\bar{f}$-core $\left(\frac{H Z(G)}{Z(G)}\right)$. Then

(i) $L$ and $\frac{G}{L}$ are free abelian groups;

(ii) $G=H H^{f}$;

(iii) $Z_{c-1}(G) \leq L$;

(iv) $Z(G)=\sqrt[\bar{G}]{\gamma_{c}(G)}$. 
Proof. Suppose that $G$ is non-abelian. Since $Z(H) \neq Z(G)$, we may suppose that $[G: H Z(G)]=p,[H Z(G): H]=q$. Then $[Z(G): Z(H)]=q$ and $H Z(G)=H L$.

The subgroups $H Z(G),(H Z(G))^{\prime}=H^{\prime}$ are normal in $G$. Since $\left(\frac{G}{L}, \frac{H L}{L}, \bar{f}\right)$ is a simple triple of degree a prime, by Corollary $2, \frac{G}{L}$ is either free abelian or finite. We know that $L$ and $\sqrt[G]{L}$ are abelian. Therefore, $\frac{G}{L}$ is free abelian.

As $Z(H)^{f} \not \leq Z(H)$ and $[Z(G): Z(H)]=q$, we obtain $Z(G)=Z(H) Z(H)^{f}$. It follows from $(H Z(G)) H^{f}=H H^{f}$ that $H H^{f}$ is a subgroup of $G$ and $H H^{f}=$ $H Z(G)$ or $G$. Suppose the first alternative holds. Then $\left(H H^{f}\right)^{\prime}=H^{\prime}$, a normal subgroup of $G$ and $\left(H^{f}\right)^{\prime}=\left(H^{\prime}\right)^{f} \leq H^{\prime}$. Therefore, $H$ is abelian and hence central; this leads to $[G: Z(G)]=p$, which is absurd.

Consider the first index $j$ such that $Z_{j}(G) \not \leq L$ and define $K=Z_{j}(G) L$. We assert that $\gamma_{i}(K) \leq Z_{j-i+1}(G)$ for $i \geq 2$ :

$$
\gamma_{2}(K)=Z_{j}(G)^{\prime}\left[L, Z_{j}(G)\right] \leq Z_{j-1}(G) \leq L ;
$$

if $\gamma_{i}(K) \leq Z_{j-i+1}(G)$ for some $i$ then

$$
\begin{aligned}
\gamma_{i+1}(K) & \leq\left[Z_{j-i+1}(G), Z_{j}(G) L\right] \\
& \leq\left[Z_{j-i+1}(G), Z_{j}(G)\right]\left[Z_{j-i+1}(G), L\right] \\
& \leq Z_{j-i+2}(G) .
\end{aligned}
$$

Since $(L \cap H)^{f} \leq L$, and $\left(Z_{j}(G), Z_{j}(H), f\right)$ is a triple, we have the corresponding triple $\left(\frac{K}{L}, \frac{L \bar{Z}_{j}(H)}{L}, \bar{f}\right)$. Furthermore, as $\frac{G}{L}$ is free abelian and $\left(\frac{G}{L}, \frac{L H}{L}, \bar{f}\right)$ is simple of degree $p$, it follows that $\frac{K}{L}$ is of finite index in $\frac{G}{L}$ and therefore $K$ and $G$ have the same nilpotency class; that is, $G$ has class $j=c$. Hence, $G=Z_{c}(G)=K$ and $Z_{c-1}(G) \leq L$.

Since $(Z(G), Z(H), f)$ is a simple triple of prime degree and $\left(\gamma_{c}(G), \gamma_{c}(H), f\right)$ is a sub-triple, it follows that $\left[Z(G): \gamma_{c}(G)\right]$ is finite; hence $Z(G)=\sqrt[G]{\gamma_{c}(G)}$.

5.4. A sequence of simple triples of degree 4. The groups $R, S$ produced in Subsection 3.2 will be shown to be part of an ascending sequence of simple triples $\left(G_{n}, H_{n}, f_{n}\right)$ where $\left[G_{n}: H_{n}\right]=2^{2}, d\left(G_{n}\right)=2, s(G)=2$ and $c\left(G_{n}\right)=n$. This will prove that the nilpotency class of groups in Theorem 12 cannot have a fixed upper limit.

Let $V_{n}$ be the free $\mathbb{Z}$ module $\mathbb{Z}^{n}$ and $\left\{\varepsilon_{i} \mid 1 \leq i \leq n\right\}$ its canonical basis. Define inductively $x_{n} \in \mathrm{GL}\left(V_{n}\right)$ :

$$
x_{2}=\left(\begin{array}{ll}
1 & 1 \\
0 & 1
\end{array}\right), \quad x_{n}=\left(\begin{array}{cc}
1 & \xi_{n} \\
0 & x_{n-1}
\end{array}\right), \quad \xi_{n}=\left(2^{n-2}, 0, \ldots, 0\right)
$$

for $n \geq 3$. Then $x_{n}$ acts nilpotently and uniserially on $V_{n}$. We note that $x_{n}^{2}$ leaves invariant the submodule $W_{n}=\left\langle\varepsilon_{1}, \ldots, \varepsilon_{n-2}, \varepsilon_{n-1}+\varepsilon_{n}, 2 \varepsilon_{n}\right\rangle$, where clearly 
$\left[V_{n}: W_{n}\right]=2$. Define the semidirect product $G_{n}=V_{n}\left\langle x_{n}\right\rangle$ and its subgroup $H_{n}=$ $W_{n}\left\langle x_{n}^{2}\right\rangle$. Then $G_{n}$ is nilpotent of class $n$ and $Z\left(G_{n}\right)=\left\langle\varepsilon_{n}\right\rangle$. Also $\left[G_{n}: H_{n}\right]=2^{2}$ and $Z\left(H_{n}\right)=\left\langle 2 \varepsilon_{n}\right\rangle$. Furthermore, $G_{2} \cong R, G_{3} \cong S$.

We construct inductively simple endomorphisms $f_{n}: W_{n} \rightarrow V_{n}$ :

$$
f_{2}=\left(\begin{array}{cc}
1 & \frac{1}{2} \\
0 & \frac{1}{2}
\end{array}\right), \quad f_{n}=\left(\begin{array}{cc}
2^{n-2} & \theta_{n} \\
0 & f_{n-1}
\end{array}\right), \quad \theta_{n}=\left(\theta_{n, 1}, \ldots, \theta_{n, n-1}\right)
$$

for $n \geq 3$.

We extend $f_{n}$, first by stipulating that $f_{n}: x_{n}^{2} \rightarrow x_{n}$. Then $f_{n}$ extends to a homomorphism $H_{n} \rightarrow G_{n}$ if and only if $x_{n}^{2} f_{n}=f_{n} x_{n}$. It is easy to see that $f_{2}$ satisfies this equation. For $n \geq 3, x_{n}^{2} f_{n}=f_{n} x_{n}$ is equivalent to

$$
\theta_{n}\left(1-x_{n-1}\right)=\xi_{n}\left(2^{n-2}-\left(1+x_{n-1}\right) f_{n-1}\right) .
$$

This last equation provides a unique solution $\theta_{n}$ where

$$
\begin{aligned}
\theta_{n, 1} & =2^{2} \theta_{n-1,1}+2^{2 n-6}, \\
\theta_{n, i} & =2^{i+1}\left(\theta_{n-1, i}+2^{n-4} \theta_{n-2, i-1}\right) \quad\left(2 \leq i \leq\left[\frac{n}{2}\right]\right) \\
& =0 \quad\left(\left\lfloor\frac{n+1}{2}\right\rfloor \leq i \leq n-1\right) .
\end{aligned}
$$

The referee provided the following explicit solution

$$
\theta_{n, i}=\frac{n}{i}\left(\begin{array}{c}
n-i-1 \\
i-1
\end{array}\right) 2^{\left(n-\frac{i}{2}-3\right)(i+1)+1} .
$$

The first few vectors are

$$
\theta_{3}=(3,0), \quad \theta_{4}=\left(2^{4}, 2^{2}, 0\right), \quad \theta_{5}=\left(2^{4} 5,2^{4} 5,0,0\right), \quad \theta_{6}=\left(2^{7} 3,2^{7} 3^{2}, 2^{8}, 0,0\right) .
$$

Finally, the resulting $f_{n}$ is simple. For otherwise, if $K$ is a non-trivial subgroup of $H_{n}$ which is normal in $G_{n}$ and is invariant under $f_{n}$, then $\{e\} \neq Z\left(H_{n}\right) \cap K$ would also be $f_{n}$-invariant.

\section{Index theorem and an application}

Let $G$ be a $\mathfrak{I}_{c}$-group, $H$ a subgroup of finite index $m$ and $f: H \rightarrow G$ simple. Then $\left[G: H^{f}\right]=m^{\prime},\left[\gamma_{c}(G): \gamma_{c}(H)\right]=l,\left[\gamma_{c}(G): \gamma_{c}\left(H^{f}\right)\right]=l^{\prime}$ are finite. The following general result establishes a simple arithmetic relation between $m, m^{\prime}, l, l^{\prime}$.

Theorem 14. Let $G$ be finitely generated nilpotent group, let $H$ be a subgroup of $G$ with $[G: H]=m$ finite and let $f: H \rightarrow G$ be a monomorphism. Let $\left[G: H^{f}\right]=$ $m^{\prime}$. Furthermore, let $U$ be a subgroup of $H$ and write $V=\left\langle U, U^{f}\right\rangle$. Suppose that $[V: U]=l,\left[V: U^{f}\right]=l^{\prime}$ are finite. Then there exist integers $m_{1}\left|m, m_{1}^{\prime}\right| m^{\prime}$ such that $l m_{1}^{\prime}=l^{\prime} m_{1}$. 
Before giving the proof, we note that the theorem is clearly true for finite groups, since $|H|=\left|H^{f}\right|,|U|=\left|U^{f}\right|$. However, the following example shows that the theorem is not valid for the class of 2-generated metabelian groups.

Example 7. Let $p$ be a prime number and $P$ be the subgroup of the additive rationals generated by $\left\{p^{i} \mid i \in \mathbb{Z}\right\}$. Then $P$ admits the automorphism $f: p^{i} \rightarrow p^{i-1}$. Define $G$ to be the extension of $P$ by $\langle f\rangle$; then $G$ is generated by $\{1, f\}$. Now let $H=G$ and $U=\langle p\rangle \leq P$. Then $U^{f}=\langle 1\rangle=V$. Moreover, the indices are $[G: H]=1=\left[G: H^{f}\right],[V: U]=p,\left[V: U^{f}\right]=1$.

Proof. I. Suppose $G$ is a free additive abelian group.

Then

$$
\sqrt[G]{V}=\sqrt[G]{U}=\sqrt[G]{U^{f}}
$$

and $[\sqrt[G]{U}: U]=t,\left[\sqrt[G]{U^{f}}: U^{f}\right]=t^{\prime}$ are finite. Indeed, $t=t^{\prime}$ since $f$ induces an isomorphism between the quotient groups $\frac{\sqrt[H]{U}}{U}, \frac{H^{f} \sqrt{U^{f}}}{U^{f}}$.

Define the indices

$$
[H+\sqrt[G]{V}: H]=m_{1}, \quad\left[H^{f}+\sqrt[G]{V}: H^{f}\right]=m_{1}^{\prime}
$$

where $m_{1} \mid m$ and $m_{1}^{\prime} \mid m^{\prime}$. Since

$$
H \cap \sqrt[G]{V}=\sqrt[H]{U}, \quad H^{f} \cap \sqrt[G]{V}=\sqrt[H^{f}]{U^{f}}
$$

we conclude that

$$
[\sqrt[G]{V}: \sqrt[H]{U}]=m_{1}, \quad\left[\sqrt[G]{V}: H^{f} \sqrt{U^{f}}\right]=m_{1}^{\prime} .
$$

Now we calculate the index

$$
\begin{aligned}
{\left[\sqrt[G]{V}: U \cap U^{f}\right] } & =[\sqrt[G]{V}: \sqrt[H]{U}][\sqrt[H]{U}: U]\left[U: U \cap U^{f}\right] \\
& =\left[\sqrt[G]{V}: \sqrt[H^{f}]{U^{f}}\right]\left[\sqrt[H^{f}]{U^{f}}: U^{f}\right]\left[U^{f}: U \cap U^{f}\right] .
\end{aligned}
$$

Thus $m_{1} t l^{\prime}=m_{1}^{\prime} t l$ and we reach $m_{1} l^{\prime}=m_{1}^{\prime} l$.

We have $\operatorname{Tor}(H)^{f}=\operatorname{Tor}\left(H^{f}\right) \leq \operatorname{Tor}(G)$. The map $\bar{f}: \frac{\operatorname{Tor}(G) H}{\operatorname{Tor}(G)} \rightarrow \frac{G}{\operatorname{Tor}(G)}$ where $\operatorname{Tor}(G) h \rightarrow \operatorname{Tor}(G) h^{f}$ is a well-defined monomorphism. Since $\frac{G}{\operatorname{Tor}(G)}$ is torsion-free nilpotent, it follows that $\left[\frac{G}{\operatorname{Tor}(G)}: \frac{\operatorname{Tor}(G) H^{f}}{\operatorname{Tor}(G)}\right]$ is finite and therefore $\left[G: H^{f}\right]=m^{\prime}$ is finite.

II. Suppose that $\operatorname{Tor}(G)=\{e\}$. We proceed by induction on the nilpotency class of $G$. The case where $G$ is free-abelian was done in part I. 
We introduce the following notation:

$$
\begin{gathered}
Z_{H}=H \cap Z(G), \quad Z_{H^{f}}=H^{f} \cap Z(G), \\
{[G: H Z]=m_{2}, \quad[H Z: H]=m_{1}, \quad\left[G: H^{f} Z\right]=m_{2}^{\prime}, \quad\left[H^{f} Z: H^{f}\right]=m_{1}^{\prime} .}
\end{gathered}
$$

Then

$$
\begin{gathered}
Z(H)^{f}=(H \cap Z(G))^{f}=\left(Z_{H}\right)^{f}, \\
{\left[Z: Z_{H}\right]=m_{1}, \quad\left[Z: Z_{H^{f}}\right]=m_{1}^{\prime}, \quad m=m_{2} m_{1}, \quad m^{\prime}=m_{2}^{\prime} m_{1}^{\prime} .}
\end{gathered}
$$

Similarly, with respect to $V$, denote

and

$$
Z_{V}=V \cap Z, \quad Z_{U}=U \cap Z, \quad Z_{U^{f}}=U^{f} \cap Z,
$$

$$
\left[V: U Z_{V}\right]=l_{2}, \quad\left[U Z_{V}: U\right]=l_{1}, \quad\left[V: U^{f} Z_{V}\right]=l_{2}^{\prime}, \quad\left[U^{f} Z_{V}: U^{f}\right]=l_{1}^{\prime} .
$$

Then

$$
\left[Z_{V}, Z_{U}\right]=l_{1}, \quad\left[Z_{V}, Z_{U^{f}}\right]=l_{1}^{\prime}, \quad l=l_{2} l_{1}, \quad l^{\prime}=l_{2}^{\prime} l_{1}^{\prime} .
$$

We claim that $\left(Z_{U}\right)^{f}=Z_{U}$. This follows from

$$
\left(Z_{U}\right)^{f} \leq Z\left(H^{f}\right) \leq Z(G), \quad\left(Z_{U}\right)^{f} \leq U^{f} \cap Z(G)=Z_{U^{f}}
$$

and from

$$
\begin{aligned}
& Z_{U^{f}} \leq Z\left(H^{f}\right)=Z(H)^{f}=(Z(G) \cap H)^{f} \\
& Z_{U^{f}} \leq(Z(G) \cap H)^{f} \cap U^{f}=(Z(G) \cap U)^{f}=\left(Z_{U}\right)^{f} .
\end{aligned}
$$

From the configuration

$$
Z \geq Z_{H}, \quad Z_{H^{f}}, \quad Z_{V} \geq Z_{U}, \quad Z_{U^{f}}
$$

we obtain that there exist $m_{11}\left|m_{1}, m_{11}^{\prime}\right| m_{1}^{\prime}$ such that $l_{1} m_{11}^{\prime}=l_{1}^{\prime} m_{11}$.

Next we apply induction to the nilpotency class of $G$.

We have that $Z(H) \leq Z(G)$ and $Z(H)^{f}=Z\left(H^{f}\right) \leq Z(G)$. Moreover, $\bar{f}: \frac{Z(G) H}{Z(G)} \rightarrow \frac{G}{Z(G)}$ defined by $Z(G) h \rightarrow Z(G) h^{f}$ is a monomorphism and $\left(\frac{Z(G) U}{Z(G)}\right)^{\bar{f}}=\frac{Z(G) U^{f}}{Z(G)}$. By applying induction to the class of $\frac{G}{Z(G)}$, we obtain that there exist $m_{21}\left|m_{2}, m_{21}^{\prime}\right| m_{2}^{\prime}$ such that $l_{2} m_{21}^{\prime}=l_{2}^{\prime} m_{21}$. Hence, putting together the two equations $l_{1} m_{11}^{\prime}=l_{1}^{\prime} m_{11}, l_{2} m_{21}^{\prime}=l_{2}^{\prime} m_{21}$, we obtain

$$
\begin{gathered}
m_{1}^{\prime}=m_{11}^{\prime} m_{21}^{\prime}\left|m^{\prime}, \quad m_{1}=m_{11} m_{21}\right| m, \\
l_{1} m_{11}^{\prime} l_{2} m_{21}^{\prime}=l_{1}^{\prime} m_{11} l_{2}^{\prime} m_{21}, \quad l m_{1}^{\prime}=l^{\prime} m_{1} .
\end{gathered}
$$

III. Now we argue the general case where $T=\operatorname{Tor}(G)$ is not necessarily trivial. 
Similar to the work done in part II, we define $T_{H}=T \cap H$ and likewise we define $T_{H^{f}}, T_{V}, T_{U}, T_{U^{f}}$, all finite groups. We note also that $\left(T_{U}\right)^{f}=T_{U^{f}}$ and therefore $T_{U}, T_{U^{f}}$ have equal orders. Then it follows that

$$
\left[V: U T_{V}\right]=\left[T_{V}: T_{U}\right]=\left[T_{V}: T_{U f}\right]=\left[V: U^{f} T_{V}\right] .
$$

Finally, the argument continues as in part II with $T$ substituting $Z$.

A special case of the above result is

Corollary 3. Maintain the hypotheses of the theorem.

(i) If $U^{f} \leq U$ then $l=\left[U: U^{f}\right]$ is finite and $l \mid m$.

(ii) If $U \leq U^{f}$ then $l^{\prime}=\left[U^{f}: U\right]$ is finite and $l^{\prime} \mid m^{\prime}$.

Proof. Suppose $U^{f} \leq U$. Then as $U^{f}$ is isomorphic to $U$, we conclude that $\left[U: U^{f}\right]$ is finite. The remaining assertions are direct.

We use the above divisibility criterion to prove

Theorem 15. Let $G$ be a $\mathfrak{\Upsilon}_{c}$-group, let $H$ be a subgroup of finite index $m$ in $G$ and $f: H \rightarrow G$ be an epimorphism. Let $\left[G: H Z_{c-1}(G)\right]=k,[Z(G): Z(H)]=q$. Then $\left[\gamma_{c}(G): \gamma_{c}(H)\right]$ is a $k$-number which divides $q$. If $f$ is simple and $m$ a squarefree integer then $G$ is abelian.

Proof. We may assume $c \geq 2$. By Subsection 3.1, item I.2, if $G=H Z_{c-1}(G)$ then $\gamma_{c}(G)=\gamma_{c}(H)$. So suppose that $G \neq H Z_{c-1}(G)$ and consider the free abelian group $\bar{G}=\frac{G}{Z_{c-1}(G)}$. There exist $a_{1}, a_{2}, \ldots, a_{s} \in G$ such that

$$
Z_{c-1}(G) a_{1}, Z_{c-1}(G) a_{2}, \ldots, Z_{c-1}(G) a_{s}
$$

freely generate $\bar{G}$ and integers $k_{1}\left|k_{2}\right| \ldots \mid k_{r}, k=k_{1} k_{2} \ldots k_{r}$ such that

$$
Z_{c-1}(G) a_{1}^{k_{1}}, Z_{c-1}(G) a_{2}^{k_{2}}, \ldots, Z_{c-1}(G) a_{r}^{k_{r}}, Z_{c-1}(G) a_{r+1}, \ldots, Z_{c-1}(G) a_{s}
$$

freely generate $\bar{H}=\frac{H Z_{c-1}(G)}{Z_{c-1}(G)}$. Thus there exist $c_{1}, c_{2}, \ldots, c_{s} \in Z_{c-1}(G)$ with

$$
H=\left\langle c_{1} a_{1}^{k_{1}}, c_{2} a_{2}^{k_{2}}, \ldots, c_{r} a_{r}^{k_{r}}, c_{r+1} a_{r+1}, \ldots, c_{s} a_{s}\right\rangle Z_{c-1}(H) .
$$

Now $\gamma_{c}(G) \leq Z(G)$ is generated by simple commutators $\left[a_{i_{1}}, a_{i_{2}}, \ldots, a_{i_{c}}\right]$ of weight $c$ where the indices $i_{j}$ are from $\{1,2, \ldots, s\}$. Whereas, $\gamma_{c}(H)$ is generated by $\left[a_{i_{1}}, a_{i_{2}}, \ldots, a_{i_{c}}\right]^{\lambda\left(i_{1}, \ldots, i_{c}\right)}$ where $\lambda\left(i_{1}, \ldots, i_{c}\right)=k_{1}^{u_{1}} \ldots k_{t}^{u_{t}} \neq 1$ and $u_{z}$ is the number of $i_{j}=z \in\{1, \ldots, r\}$. Therefore, $\left|\frac{\gamma_{c}(G)}{\gamma_{c}(H)}\right|$ is a $k$-number.

As $f$ induces epimorphisms $\gamma_{c}(H) \rightarrow \gamma_{c}(G), Z(H) \rightarrow Z(G)$, we apply Corollary 3 to obtain $\left|\frac{\gamma_{c}(G)}{\gamma_{c}(H)}\right|$ divides $\left|\frac{Z(G)}{Z(H)}\right|=q$. 
Now suppose that $f$ is simple, $m$ is square-free and $G$ is non-abelian. Since $f$ is simple, we have $Z_{c-1}(G) \not \leq H$. Let

$$
\left[G, H Z_{c-1}(G)\right]=m_{1}, \quad\left[H Z_{c-1}(G), H\right]=m_{2} ;
$$

then $\operatorname{gcd}\left(m_{1}, m_{2}\right)=1$. Since $[Z(G): Z(H)]$ divides $m_{2}$, we conclude that $\gamma_{c}(G)=$ $\gamma_{c}(H)$ and hence $\gamma_{c}(G)=\{e\}$, a contradiction.

\section{References}

[1] S. V. Aleshin, A free group of finite automata. Vestnik Moskov. Univ. Ser. I Mat. Mekh. 1983, 12-14; Moscow Univ. Math. Bull. 38 (1983), 10-13. Zbl 0522.68054 MR 0713968

[2] A. M. Brunner and S. Sidki, The generation of $\operatorname{GL}(n, \mathbb{Z})$ by finite automata. Internat. J. Algebra Comput. 8 (1998), 127-139. Zbl 0923.20023 MR 1492064

[3] I. Belegradek, On co-hopfian nilpotent groups. Bull. London Math. Soc. 35 (2003), 805-811. Zbl 1042.20022 MR 2000027

[4] Y. Glasner and S. Mozes, Automata and square complexes. Geom. Dedicata 111 (2005), 43-64. Zbl 1088.20037 MR 2155175

[5] P. Hall, Nilpotent groups. Queen Mary College Mathematics Notes, London 1969. Zbl 0211.34201 MR 0283083

[6] V. Nekrashevych, Self-similar groups. Math. Surveys Monogr. 117, Amer. Math. Soc., Providence, RI, 2005. Zbl 1087.20032 MR 2162164

[7] V. Nekrashevych and S. Sidki, Automorphisms of the binary tree: state-closed subgroups and dynamics of 1/2-endomorphisms. In Groups, topological, combinatorial and arithmetic aspects, London Math. Lecture Note Ser. 311, Cambridge University Press, Cambridge 2004, 375-404. Zbl 02152960 MR 2073355

[8] D. Segal, Polycyclic groups. Cambridge University Press, Cambridge 1983. Zbl 0516.20001 MR 0713786

[9] M. Shub, Endomorphisms of compact differentiable manifolds. Amer. J. Math. 91 (1969), 175-199. Zbl 0201.56305 MR 0240824

[10] S. Sidki, Automorphisms of one-rooted trees: growth, circuit structure and acyclicity. J. Math. Sci. 100 (2000), 1925-1943. Zbl 1069.20504 MR 1774362

[11] G. C. Smith, Compressibility in nilpotent groups. Bull. London Math. Soc. 17 (1985), 453-457. Zbl 0556.20029 MR 0806011

[12] M. Vorobets and Y. Vorobets, On a free group of transformations defined by an automaton. arXive:math.GR/0601231

Received May 25, 2006; revised September 6, 2006

Departamento de Matemática, Universidade Federal de Mato Grosso, ICLMA, Pontal do Araguaia, MT, Brazil

E-mail: berlatto@cpd.ufmt.br

Departamento de Matemática, Universidade de Brasília, Brasília DF 70910-900, Brazil

E-mail: sidki@mat.unb.br. 